Supporting information

\title{
Metal-free Synthesis of $\mathrm{N}$-(Carboselenoate) benzimidazolones by Cascade Cyclization of ortho- Diisocyanoarenes and Selenosulfonates
}

\author{
Yi Fang, ${ }^{\dagger}$ Can Liu, ${ }^{\dagger}$ Weidong Rao, ${ }^{\dagger}$ Shun-Yi Wang, ${ }^{*, \dagger}$ and Shun-Jun Ji*,† \\ †Key Laboratory of Organic Synthesis of Jiangsu Province, College of Chemistry, Chemical Engineering and Materials Science, \\ and Collaborative Innovation Center of Suzhou Nano Science and Technology, Soochow University, Suzhou, 215123, China. \\ $\ddagger$ Jiangsu Key Laboratory of Biomass-based Green Fuels and Chemicals, College of Chemical Engineering, Nanjing Forestry \\ University, Nanjing 210037, China.
}


Table of Contents

General

information

2. General

procedure for

synthesis of

triazine

compounds 
3. General

procedure for

synthesis of

diazo

compounds 
4. General

Procedure for

table 2

5. General

Procedure for

table 3 


\section{Deuterium}

labelling

experiments

for Scheme 3

7. Control

experiments

for Scheme 4 


$$
\text { 8. X-ray }
$$

structure of

$3 \mathrm{~g}$ and $5 \mathrm{~d}$

9. References

10.

1

H NMR and

13 


\section{NMR}

Spectra of

\section{compounds}

1. General information.............................................. 2

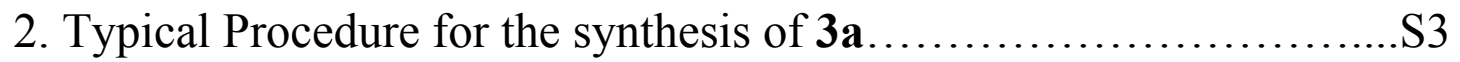

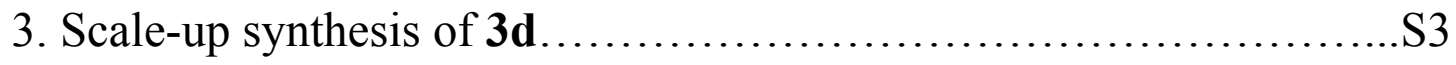

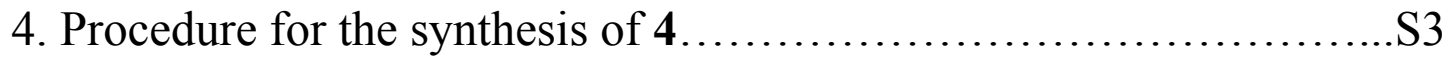

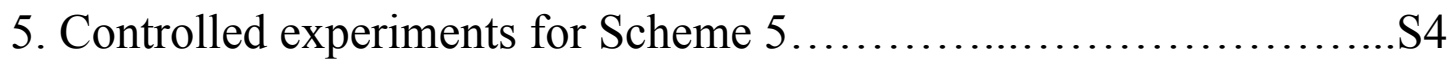

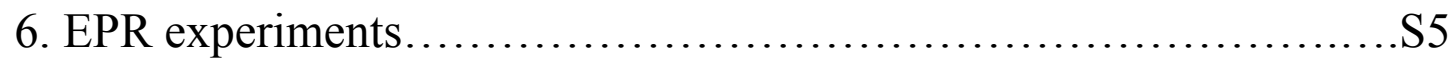

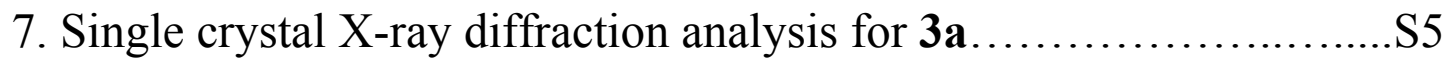

8. Spectroscopic data of compounds................................ 7

9. Copies of ${ }^{1} \mathrm{H}$ and ${ }^{13} \mathrm{C}$ NMR spectra for compounds......................S14

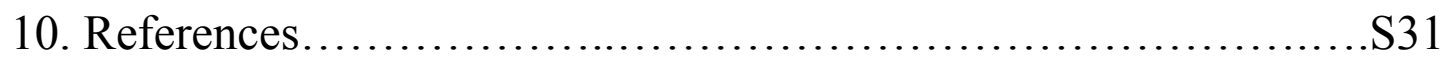




\section{General Information}

Unless otherwise noted, all commercially available compounds were used as provided without further purification. Solvents for chromatography were purchased from Adamas-beta ${ }^{\circledR}$ and used without further purification. Se-alkyl selenosulfonates were purchased from Suzhou Chukai Pharma Tech Co., Ltd. ortho-Diisocyanides were prepared according to published literature procedures, triphosgene could be employed instead of diphosgene in the preparation. ${ }^{1}$ Analytical thin-layer chromatography (TLC) was performed on silica gel, visualized by $\mathrm{I}_{2}$ or irradiation with UV light. For column chromatography, 200300 mesh silica gel was used. ${ }^{1} \mathrm{H}-\mathrm{NMR}$ and ${ }^{13} \mathrm{C}-\mathrm{NMR}$ were recorded on a BRUKER $400 \mathrm{MHz}$ spectrometer in $\mathrm{CDCl}_{3}$ or DMSO- $d_{6}$. Chemical shifts $(\delta)$ were reported referenced to an internal tetramethylsilane standard or the $\mathrm{CDCl}_{3}$ residual peak $\left(\delta\right.$ 7.26) or DMSO- $d_{6}$ residual peak $(\delta 2.50)$ for ${ }^{1} \mathrm{H}$ NMR. Chemical shifts of ${ }^{13} \mathrm{C}$ NMR are reported relative to $\mathrm{CDCl}_{3}(\delta 77.16)$ or DMSO- $d_{6}(\delta 39.52)$. Data are reported in the following order: chemical shift $(\delta)$ in ppm; multiplicities are indicated s (singlet), bs (broad singlet), d (doublet), t (triplet), m (multiplet); coupling constants ( $\mathrm{J}$ ) are in Hertz (Hz). Melting points were measured on an Electrothermal digital melting point apparatus and were uncorrected. IR spectra were recorded on a BRUKER VERTEX 70 spectrophotometer and are reported in terms of frequency of absorption $\left(\mathrm{cm}^{-1}\right)$. HRMS spectra were obtained by using BRUKER micrOTOF-Q III instrument with ESI source. ESR spectra were detected by JES-X320 electron spin resonance instrument. 


\section{Typical Procedure for the synthesis of 3a}<smiles>COc1cc2[nH]c(=O)n(C(=O)CCOc3ccccc3)c2cc1OC</smiles>

In an oven-dried screw-capped $8-\mathrm{mL}$ vial equipped with a magnetic stir bar, 1,2-diisocyano-4,5dimethylbenzene 1a ( $0.1 \mathrm{mmol}, 1.0$ equiv.) and $\mathrm{Se}$-(2-phenoxyethyl) benzenesulfonoselenoate $\mathbf{2 a}(0.2 \mathrm{mmol}$, 2.0 equiv.) were added. Subsequently, $t-\mathrm{BuOH}(2 \mathrm{~mL})$ was added. The system was stirred at $30^{\circ} \mathrm{C}$ under air. After $12 \mathrm{~h}$, the reaction was heated to $50^{\circ} \mathrm{C} .4 \mathrm{~h}$ later, the crude reaction mixture was cooled to room temperature and diluted with ethyl acetate $(100 \mathrm{~mL})$. The organic phase was washed with brine $(20 \mathrm{~mL}$ $\times 2$ ). The organic layer was dried over $\mathrm{Na}_{2} \mathrm{SO}_{4}$, filtered, and concentrated. The residue was purified by flash chromatography (EtOAc/PE $=1: 20$ to $1: 3$ ) to obtain the desired product 3a.

\section{Scale-up synthesis of $3 d$}<smiles>N#Cc1cc2ccccc2cc1N</smiles>

1d<smiles>O=S(=O)([SeH])c1ccccc1CCOc1ccccc1</smiles>

$2 a$

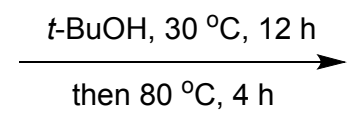<smiles>O=C([Se]CCOc1ccccc1)n1c(=O)[nH]c2cc3ccccc3cc21</smiles>

$3 d$

In an oven-dried round-bottom flask equipped with a magnetic stir bar, 2,3-diisocyanonaphthalene 1d (1.0 mmol, 1.0 equiv.) and $\mathrm{Se}$-(2-phenoxyethyl) benzenesulfonoselenoate $\mathbf{2 a}(2.0 \mathrm{mmol}, 2.0$ equiv.) were added. Subsequently, $t$-BuOH $(20 \mathrm{~mL})$ was added. The system was stirred at $30^{\circ} \mathrm{C}$ under air. After $12 \mathrm{~h}$, the reaction was heated to $80^{\circ} \mathrm{C} .4 \mathrm{~h}$ later, the crude reaction mixture was cooled to room temperature and diluted with ethyl acetate $(100 \mathrm{~mL})$. The organic phase was washed with brine $(20 \mathrm{~mL} \times 2)$. The organic layer was dried over $\mathrm{Na}_{2} \mathrm{SO}_{4}$, filtered, and concentrated. The residue was purified by flash chromatography (EtOAc/DCM $=1: 20$ to $1: 1$ ) to obtain the desired product $3 \mathbf{d}$ in $83 \%$ yield (342.2 $\mathrm{mg}$ ).

\section{Procedure for the synthesis of 4}<smiles>Cc1cc2[nH]c(=O)n(C(=O)[Se]CCOc3ccccc3)c2cc1C</smiles>

3a

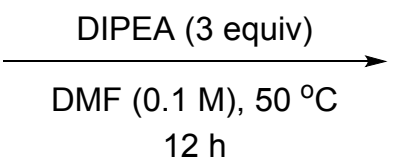

$12 \mathrm{~h}$<smiles>Cc1cc2[nH]c(=O)n(C(=O)NCc3ccccc3)c2cc1C</smiles>

$4,75 \%$

In a round-bottom flask equipped with a magnetic stir bar, 3a $(0.1 \mathrm{mmol}, 1$ equiv. $)$ was dissolved in DMF 
$(1 \mathrm{~mL})$. Subsequently, benzylamine $(0.2 \mathrm{mmol}, 2$ equiv. $)$ and DIPEA $(0.3 \mathrm{mmol}, 3$ equiv. $)$ were added and the mixture was stirred at $50{ }^{\circ} \mathrm{C}$ for $12 \mathrm{~h}$. The crude reaction mixture was cooled to room temperature and diluted with dichloromethane $(100 \mathrm{~mL})$. The organic phase was washed with brine $(20 \mathrm{~mL} \times 2)$. The organic layer was dried over $\mathrm{Na}_{2} \mathrm{SO}_{4}$, filtered, and concentrated. Then, ethyl ether was added to precipitate the desired product. The product $\mathbf{4}$ was collected by filtration and dried under vacuum.

\section{Controlled experiments for Scheme 5}

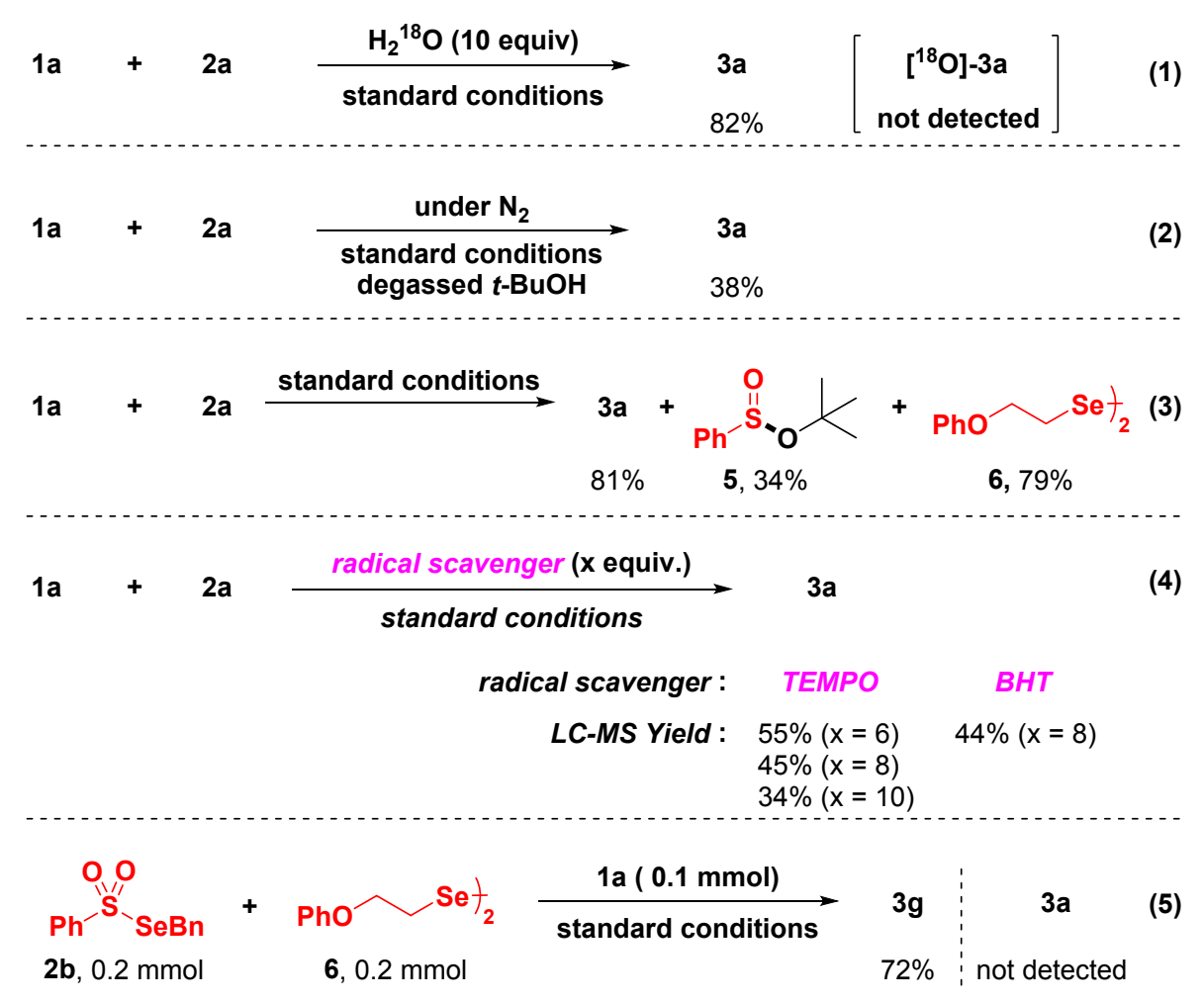

For eq. (1): In an oven-dried screw-capped 8-mL vial equipped with a magnetic stir bar, isocyanide 1a ( $0.1 \mathrm{mmol}, 1.0$ equiv.) and selenosulfonate $2 \mathrm{a}(0.2 \mathrm{mmol}, 2.0$ equiv.) were dissolved in $t$ - $\mathrm{BuOH}(2 \mathrm{~mL})$. $\mathrm{H}_{2}{ }^{18} \mathrm{O}$ (1.0 mmol, 10 equiv.) were added subsequently. The system was stirred at $30{ }^{\circ} \mathrm{C}$ under air. After $12 \mathrm{~h}$, the reaction was heated to $50{ }^{\circ} \mathrm{C} .4 \mathrm{~h}$ later, the crude reaction mixture was cooled to room temperature and diluted with ethyl acetate $(100 \mathrm{~mL})$. The organic phase was washed with brine $(20 \mathrm{~mL}$ $\times 2$ ). The organic layer was dried over $\mathrm{Na}_{2} \mathrm{SO}_{4}$, filtered, and concentrated. The residue was purified by flash chromatography (EtOAc/PE $=1: 20$ to $1: 3$ ) to obtain the desired product 3a. The product was analyzed by HRMS.

For eq. (2): In an oven-dried screw-capped 8-mL vial equipped with a magnetic stir bar, isocyanide 1a ( $0.1 \mathrm{mmol}, 1.0$ equiv.) and selenosulfonate $\mathbf{2 a}(0.2 \mathrm{mmol}, 2.0$ equiv.) were dissolved in degassed $t$ - $\mathrm{BuOH}$ $(2 \mathrm{~mL})$. The system was stirred at $30^{\circ} \mathrm{C}$ under $\mathrm{N}_{2}$. After $12 \mathrm{~h}$, the reaction was heated to $50{ }^{\circ} \mathrm{C} .4 \mathrm{~h}$ later, the crude reaction mixture was cooled to room temperature and diluted with ethyl acetate $(100 \mathrm{~mL})$. The organic phase was washed with brine $(20 \mathrm{~mL} \times 2)$. The organic layer was dried over $\mathrm{Na}_{2} \mathrm{SO}_{4}$, filtered, and concentrated. The residue was purified by flash chromatography (EtOAc/PE $=1: 20$ to $1: 3$ ) to obtain 
the desired product $\mathbf{3 a}$.

For eq. (3): In an oven-dried screw-capped 8-mL vial equipped with a magnetic stir bar, isocyanide 1a ( $0.1 \mathrm{mmol}, 1.0$ equiv.) and selenosulfonate $\mathbf{2 a}(0.2 \mathrm{mmol}, 2.0$ equiv.) were dissolved in $t$ - $\mathrm{BuOH}(2 \mathrm{~mL})$. The system was stirred at $30^{\circ} \mathrm{C}$ under air. After $12 \mathrm{~h}$, the reaction was heated to $50^{\circ} \mathrm{C} .4 \mathrm{~h}$ later, the crude reaction mixture was cooled to room temperature and diluted with ethyl acetate $(100 \mathrm{~mL})$. The organic phase was washed with brine $(20 \mathrm{~mL} \times 2)$. The organic layer was dried over $\mathrm{Na}_{2} \mathrm{SO}_{4}$, filtered, and concentrated. The residue was purified by flash chromatography to obtain the desired products 5 (PE), $6(\mathrm{PE}$ to $\mathrm{EtOAc} / \mathrm{PE}=1: 20)$ and $\mathbf{3 a}(\mathrm{EtOAc} / \mathrm{PE}=1: 20$ to $1: 3)$.

For eq. (4): In an oven-dried screw-capped 8-mL vial equipped with a magnetic stir bar, isocyanide 1a ( $0.1 \mathrm{mmol}, 1.0$ equiv.), selenosulfonate $\mathbf{2 a}(0.2 \mathrm{mmol}, 2.0$ equiv.) and radical scavenger TEMPO (or BHT) were dissolved in $t$-BuOH $(2 \mathrm{~mL})$. The system was stirred at $30{ }^{\circ} \mathrm{C}$ under air. After $12 \mathrm{~h}$, the reaction was heated to $50{ }^{\circ} \mathrm{C} .4 \mathrm{~h}$ later, the crude reaction mixture was cooled to room temperature and biphenyl $(0.1 \mathrm{mmol}, 1.0$ equiv. $)$ was added as internal standard. The mixture was diluted with ethyl acetate $(5 \mathrm{~mL})$ and stirred at $\mathrm{rt}$ for $2 \mathrm{~min}$. Then, $0.5 \mathrm{~mL}$ of the mixture was taken to a sample vial and diluted with ethyl acetate $(1.5 \mathrm{~mL})$. Subsequently, the sample was analyzed by LC-MS to determine the yield.

For eq. (5): In an oven-dried screw-capped 8-mL vial equipped with a magnetic stir bar, isocyanide 1a (0.1 mmol, 1.0 equiv.), Se-benzyl benzenesulfonoselenoate $\mathbf{2 b}(0.2 \mathrm{mmol}, 2$ equiv.) and 1,2-bis(2phenoxyethyl)diselane 6 ( $0.2 \mathrm{mmol}, 2.0$ equiv.) were dissolved in $t$ - $\mathrm{BuOH}(2 \mathrm{~mL})$. The system was stirred at $30^{\circ} \mathrm{C}$ under air. After $12 \mathrm{~h}$, the reaction was heated to $50^{\circ} \mathrm{C} .4 \mathrm{~h}$ later, the crude reaction mixture was cooled to room temperature and diluted with ethyl acetate $(100 \mathrm{~mL})$. The organic phase was washed with brine $(20 \mathrm{~mL} \times 2)$. The organic layer was dried over $\mathrm{Na}_{2} \mathrm{SO}_{4}$, filtered, and concentrated. The residue was purified by flash chromatography (EtOAc/PE $=1: 20$ to $1: 3$ ) to obtain the desired product $\mathbf{3 g}$.

\section{EPR experiments}

EPR Studies of Interaction between 1a and 2a. Three dried tubes equipped with a stir bar were respectively loaded with (1) DMPO + $\mathbf{1 a}$ in $t-\mathrm{BuOH}$; (2) $\mathrm{DMPO}+\mathbf{2 a}$ in $t-\mathrm{BuOH} ;$ (3) $\mathrm{DMPO}+\mathbf{1 a}+\mathbf{2 a}$ in $t$ - $\mathrm{BuOH}$. They were all stirred at $50^{\circ} \mathrm{C}$. After $10 \mathrm{~min}$, the solution samples were taken by four $0.3 \mathrm{~mm}$ glass capillaries and analyzed by EPR. EPR spectra was recorded at room temperature on EPR spectrometer operated at $9146.941 \mathrm{MHz}$. Typical spectrometer parameters are shown as follows, Mod freq $=100 \mathrm{kHz}$, Field center $=326.000 \mathrm{mT}$, width $=+/-6.000 \mathrm{mT}$, Sweep time $=30 \mathrm{~s}$.

\section{Single crystal X-ray diffraction analysis for 3a.}

The X-ray crystallographic coordinates for structure reported in this article have been deposited at the Cambridge Crystallographic Data Centre (CCDC), under deposition number CCDC 1904577 (3a). These data can be obtained free of charge from The Cambridge Crystallographic Data Centre via http://www.ccdc.cam.ac.uk. 
<smiles>C#C[Se]c1ccccc1CC[Se]C(=O)n1c(=O)[nH]c2cc(C)c(C)cc21</smiles>

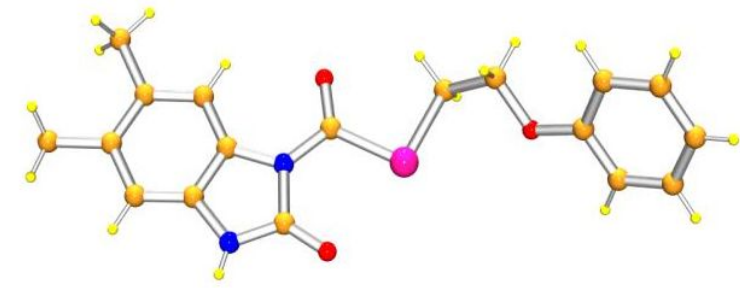




\section{Spectroscopic data of compounds}<smiles>Cc1cc2[nH]c(=O)n(C(=O)[Se]CCOc3ccccc3)c2cc1C</smiles>

$S e$-(2-phenoxyethyl) 5,6-dimethyl-2-oxo-2,3-dihydro-1 $H$-benzo $[d]$ imidazole-1-carboselenoate (3a) White solid (31.5 mg, 81\%). Mp: 168.5-169.8 ${ }^{\circ} \mathrm{C} .{ }^{1} \mathbf{H}$ NMR (400 MHz, DMSO- $\left.d_{6}\right) \delta 11.52(\mathrm{~s}, 1 \mathrm{H}), 7.76$ (s, 1H), $7.32-7.25(\mathrm{~m}, 2 \mathrm{H}), 6.99-6.91(\mathrm{~m}, 3 \mathrm{H}), 6.87(\mathrm{~s}, 1 \mathrm{H}), 4.23(\mathrm{t}, J=6.9 \mathrm{~Hz}, 2 \mathrm{H}), 3.23(\mathrm{t}, J=7.0$ $\mathrm{Hz}, 2 \mathrm{H}), 2.23$ (s, 6H). ${ }^{13} \mathbf{C}$ NMR (100 MHz, DMSO- $\left.d_{6}\right) \delta 164.3,158.1,152.4,133.0,129.8,129.5,126.4$, 124.2, 120.7, 114.8, 114.5, 110.5, 66.8, 23.3, 19.6, 19.5. IR (neat): $v=3205,2924,2859,1723,1675$, 1344, $1250 \mathrm{~cm}^{-1}$. HRMS (ESI): calcd. for $\mathrm{C}_{18} \mathrm{H}_{18} \mathrm{~N}_{2} \mathrm{O}_{3} \mathrm{SeNa}$ [M+Na] $]^{+}: 413.0375$, found: 413.0387 .<smiles>COc1cc2[nH]c(=O)n(C(=O)[Se]CCc3ccccc3)c2cc1OC</smiles>

$S e$-(2-phenoxyethyl) $\quad 5,6$-dimethoxy-2-oxo-2,3-dihydro-1 $H$-benzo $[d]$ imidazole-1-carboselenoate (3b)

White solid (26.5 mg, 63\%). Mp: 202.1-203.5 ${ }^{\circ} \mathrm{C} .{ }^{1} \mathbf{H}$ NMR (400 MHz, DMSO- $\left.d_{6}\right) \delta 11.50(\mathrm{~s}, 1 \mathrm{H}), 7.62$ (s, 1H), $7.33-7.24(\mathrm{~m}, 2 \mathrm{H}), 6.94$ (dd, $J=15.9,7.8 \mathrm{~Hz}, 3 \mathrm{H}), 6.71(\mathrm{~s}, 1 \mathrm{H}), 4.23(\mathrm{t}, J=6.9 \mathrm{~Hz}, 2 \mathrm{H}), 3.77$ (s, 3H), 3.74 (s, 3H), 3.24 (t, $J=6.9 \mathrm{~Hz}, 2 \mathrm{H}) .{ }^{13} \mathrm{C}$ NMR (100 MHz, DMSO-d $\left.d_{6}\right) \delta$ 164.4, 158.1, 152.6, 147.0, 144.3, 129.6, 121.8, 120.8, 118.8, 114.5, 100.3, 95.2, 66.8, 56.3, 56.0, 23.4. IR (neat): $v=3115$, 2924, 2853, 1702, $1492 \mathrm{~cm}^{-1}$. HRMS (ESI): calcd. for $\mathrm{C}_{18} \mathrm{H}_{18} \mathrm{~N}_{2} \mathrm{O}_{5} \mathrm{SeNa}[\mathrm{M}+\mathrm{Na}]^{+}: 445.0273$, found: 445.0263.<smiles>O=C([SeH2]CCOc1ccccc1)n1c(=O)[nH]c2cc(Br)c(Br)cc21</smiles>

Se-(2-phenoxyethyl) 5,6-dibromo-2-oxo-2,3-dihydro-1H-benzo[d] imidazole-1-carboselenoate (3c) Yellow solid (37.4 mg, 70\%). Mp: 229.1-230.5 ${ }^{\circ} \mathrm{C} .{ }^{1} \mathbf{H}$ NMR (400 MHz, DMSO- $\left.d_{6}\right) \delta 11.98(\mathrm{~s}, 1 \mathrm{H})$, $8.16(\mathrm{~s}, 1 \mathrm{H}), 7.38(\mathrm{~s}, 1 \mathrm{H}), 7.32-7.25(\mathrm{~m}, 2 \mathrm{H}), 6.94(\mathrm{dd}, J=13.6,7.6 \mathrm{~Hz}, 3 \mathrm{H}), 4.24(\mathrm{t}, J=6.8 \mathrm{~Hz}, 2 \mathrm{H})$, 
$3.26(\mathrm{t}, J=6.9 \mathrm{~Hz}, 2 \mathrm{H}) .{ }^{13} \mathrm{C}$ NMR $\left(100 \mathrm{MHz}, \mathrm{DMSO}-d_{6}\right) \delta 165.0,158.1,152.0,129.5,129.4,126.6$, $120.8,118.9,118.0,115.5,114.5,114.0,66.6,23.7$. IR (neat): $v=3115,2915,2860,1733,1664,1329$, $1062 \mathrm{~cm}^{-1}$. HRMS (ESI): calcd. for $\mathrm{C}_{16} \mathrm{H}_{12} \mathrm{Br}_{2} \mathrm{~N}_{2} \mathrm{O}_{3} \mathrm{SeNa}[\mathrm{M}+\mathrm{Na}]^{+}:$542.8252, found: 542.8270 .<smiles>O=C([Se]CCOc1ccccc1)n1c(=O)[nH]c2cc3ccccc3cc21</smiles>

Se-(2-phenoxyethyl) 2-oxo-2,3-dihydro-1H-naphtho[2,3- $d]$ imidazole-1-carboselenoate (3d) White solid (37.4 mg, 91\%). Mp: 230.8-231.6 ${ }^{\circ} \mathrm{C} .{ }^{1} \mathbf{H}$ NMR (400 MHz, DMSO-d $) \delta 11.91$ (s, $\left.1 \mathrm{H}\right), 8.46$ (s, 1H), $7.94(\mathrm{~d}, J=8.0 \mathrm{~Hz}, 1 \mathrm{H}), 7.89(\mathrm{~d}, J=8.1 \mathrm{~Hz}, 1 \mathrm{H}), 7.49-7.38(\mathrm{~m}, 3 \mathrm{H}), 7.33-7.27(\mathrm{~m}, 2 \mathrm{H}), 7.01$ $-6.92(\mathrm{~m}, 3 \mathrm{H}), 4.27(\mathrm{t}, J=6.9 \mathrm{~Hz}, 2 \mathrm{H}), 3.29(\mathrm{t}, J=6.9 \mathrm{~Hz}, 2 \mathrm{H}) .{ }^{13} \mathbf{C}$ NMR $\left(100 \mathrm{MHz}, \mathrm{DMSO}-d_{6}\right) \delta$ $164.4,158.1,152.9,130.8,129.6,129.3,128.5,128.2,127.0,126.8,125.6,124.4,120.8,114.5,111.3$, 105.1, 66.8, 23.6. IR (neat): $v=3199,3159,1734,1674,1204,1041 \mathrm{~cm}^{-1}$. HRMS (ESI): calcd. for $\mathrm{C}_{20} \mathrm{H}_{16} \mathrm{~N}_{2} \mathrm{O}_{3} \mathrm{SeNa}[\mathrm{M}+\mathrm{Na}]^{+}:$435.0218, found: 435.0203 .<smiles>Cc1cccc2c1[nH]c(=O)n2C(=O)[Se]CCOc1ccccc1</smiles><smiles>Cc1cccc2[nH]c(=O)n(C(=O)[SeH])c12</smiles>

$S e$-(2-phenoxyethyl) 7-methyl-2-oxo-2,3-dihydro-1H-benzo[d]imidazole-1-carboselenoate (3e) Se-(2-phenoxyethyl) 4-methyl-2-oxo-2,3-dihydro-1H-benzo[ $d]$ imidazole-1-carboselenoate (3e') White solid (26.4 mg, 70\%). Mp: 189.4-190.7 ${ }^{\circ} \mathrm{C} .{ }^{1} \mathbf{H}$ NMR (400 MHz, DMSO- $\left.d_{6}\right) \delta 11.74(\mathrm{~s}, 1 \mathrm{H}), 11.65$ (s, $0.70 \mathrm{H}), 7.79(\mathrm{dd}, J=6.6,2.5 \mathrm{~Hz}, 1 \mathrm{H}), 7.31-7.26(\mathrm{~m}, 3.42 \mathrm{H}), 7.13(\mathrm{t}, J=7.8 \mathrm{~Hz}, 0.73 \mathrm{H}), 7.05-$ $7.02(\mathrm{~m}, 1.8 \mathrm{H}), 7.00-6.87$ (m, $7.13 \mathrm{H}), 4.26-4.22(\mathrm{~m}, 3.4 \mathrm{H}), 3.26-3.20(\mathrm{~m}, 3.4 \mathrm{H}), 2.40(\mathrm{~s}, 2.1 \mathrm{H})$, $2.30(\mathrm{~s}, 3 \mathrm{H}) .{ }^{13} \mathrm{C}$ NMR $\left(100 \mathrm{MHz}, \mathrm{DMSO}-d_{6}\right) \delta 164.8,164.6,158.1,152.9,152.7,129.9,129.5,127.3$, $126.1,125.8,125.5,125.4,125.2,125.2,122.0,120.7,119.5,114.5,111.4,107.4,66.8,24.2,23.4,21.8$, 16.1. IR (neat): $v=3159,3064,2925,2858,1726,1674,1599,1495,1344 \mathrm{~cm}^{-1}$. HRMS (ESI): calcd. for $\mathrm{C}_{17} \mathrm{H}_{16} \mathrm{~N}_{2} \mathrm{O}_{3} \mathrm{SeNa}[\mathrm{M}+\mathrm{Na}]^{+}$: 399.0218 , found: 399.0222 . 
<smiles>CC(=O)c1ccc2c(c1)[nH]c(=O)n2C(=O)[Se]CCOc1ccccc1</smiles>

methyl 2-oxo-3-(((2-phenoxyethyl)selanyl)carbonyl)-2,3-dihydro-1H-benzo[d]imidazole-5-carboxylate (3f) White solid (15.8 mg, 37\%). Mp: $190.4-191.7^{\circ} \mathrm{C} .{ }^{1} \mathbf{H}$ NMR (400 MHz, DMSO- $\left.d_{6}\right) \delta 11.93$ (s, 1H), 8.04 $(\mathrm{d}, J=8.4 \mathrm{~Hz}, 1 \mathrm{H}), 7.76(\mathrm{dd}, J=8.4,1.7 \mathrm{~Hz}, 1 \mathrm{H}), 7.55(\mathrm{~s}, 1 \mathrm{H}), 7.32-7.24(\mathrm{~m}, 2 \mathrm{H}), 6.95(\mathrm{dd}, J=16.1$, $7.8 \mathrm{~Hz}, 3 \mathrm{H}), 4.25(\mathrm{t}, J=6.9 \mathrm{~Hz}, 2 \mathrm{H}), 3.85(\mathrm{~s}, 3 \mathrm{H}), 3.27(\mathrm{t}, J=6.9 \mathrm{~Hz}, 2 \mathrm{H}) .{ }^{13} \mathbf{C}$ NMR $(100 \mathrm{MHz}$, DMSO$\left.d_{6}\right) \delta 165.7,165.1,158.1,152.4,129.7,129.6,128.7,125.9,123.8,120.8,114.5,113.8,109.9,66.6,52.3$, 23.6. IR (neat): $v=3205,1732,1717,1683,1457,1292,1057 \mathrm{~cm}^{-1}$. HRMS (ESI): calcd. for $\mathrm{C}_{18} \mathrm{H}_{16} \mathrm{~N}_{2} \mathrm{O}_{5} \mathrm{SeNa}[\mathrm{M}+\mathrm{Na}]^{+}: 443.0117$, found: 443.0117.<smiles>CC(=O)c1ccc2[nH]c(=O)n(C(=O)[Se]CCOc3ccccc3)c2c1</smiles>

methyl 2-oxo-1-(((2-phenoxyethyl)selanyl)carbonyl)-2,3-dihydro-1H-benzo[ $d$ ]imidazole-5-carboxylate (3f') White solid (18.2 mg, 43\%). Mp: 205.2-206.6 ${ }^{\circ} \mathrm{C} .{ }^{1} \mathbf{H}$ NMR (400 MHz, DMSO- $\left.d_{6}\right) \delta 12.08$ (s, $\left.1 \mathrm{H}\right), 8.50$ (s, 1H), $7.87(\mathrm{dd}, J=8.2,1.7 \mathrm{~Hz}, 1 \mathrm{H}), 7.32-7.26(\mathrm{~m}, 2 \mathrm{H}), 7.17(\mathrm{~d}, J=8.2 \mathrm{~Hz}, 1 \mathrm{H}), 6.95(\mathrm{dd}, J=16.4$, $7.9 \mathrm{~Hz}, 3 \mathrm{H}), 4.26(\mathrm{t}, J=6.8 \mathrm{~Hz}, 2 \mathrm{H}), 3.84(\mathrm{~s}, 3 \mathrm{H}), 3.27(\mathrm{t}, J=6.8 \mathrm{~Hz}, 2 \mathrm{H}) .{ }^{13} \mathbf{C}$ NMR $(100 \mathrm{MHz}$, DMSO$\left.d_{6}\right) \delta 165.9,165.0,158.1,152.5,132.7,129.6,126.9,126.1,123.2,120.8,114.6,114.5,109.6,66.7,52.1$, 23.7. IR (neat): $v=3241,2945,1750,1684,1327,1236 \mathrm{~cm}^{-1}$. HRMS (ESI): calcd. for $\mathrm{C}_{18} \mathrm{H}_{16} \mathrm{~N}_{2} \mathrm{O}_{5} \mathrm{SeNa}$ $[\mathrm{M}+\mathrm{Na}]^{+}:$443.0117, found: 443.0117<smiles>Cc1cc2[nH]c(=O)n(C(=O)[Se]Cc3ccccc3)c2cc1C</smiles>

Se-benzyl 5,6-dimethyl-2-oxo-2,3-dihydro-1H-benzo[d] imidazole-1-carboselenoate (3g) Off-white solid (25.0 mg, 70\%). Mp: 226.4-227.9 ${ }^{\circ} \mathrm{C} .{ }^{1} \mathbf{H}$ NMR (400 MHz, DMSO- $\left.d_{6}\right) \delta 11.48(\mathrm{~s}, 1 \mathrm{H})$, $7.75(\mathrm{~s}, 1 \mathrm{H}), 7.38(\mathrm{~d}, J=7.1 \mathrm{~Hz}, 2 \mathrm{H}), 7.29(\mathrm{t}, J=7.5 \mathrm{~Hz}, 2 \mathrm{H}), 7.20(\mathrm{t}, J=7.4 \mathrm{~Hz}, 1 \mathrm{H}), 6.84(\mathrm{~s}, 1 \mathrm{H})$, 4.15 (s, 2H), $2.22(\mathrm{~s}, 3 \mathrm{H}), 2.21(\mathrm{~s}, 3 \mathrm{H}) .{ }^{13} \mathrm{C}$ NMR (100 MHz, DMSO-d $\left.)\right) \delta 164.6,152.4,139.1,133.0$ $129.8,129.0,128.5,126.6,126.5,124.3,114.8,110.5,28.2,19.6,19.5$. IR (neat): $v=3166,2923,1723$, 
1698, 1344, $1039 \mathrm{~cm}^{-1}$. HRMS (ESI): calcd. for $\mathrm{C}_{17} \mathrm{H}_{16} \mathrm{~N}_{2} \mathrm{O}_{2} \mathrm{SeNa}[\mathrm{M}+\mathrm{Na}]^{+}$: 383.0269 , found: 383.0253 .<smiles>Cc1ccc(C[Se]C(=O)n2c(=O)[nH]c3cc(C)c(C)cc32)cc1</smiles>

$S e$-(4-methylbenzyl) 5,6-dimethyl-2-oxo-2,3-dihydro-1H-benzo[d]imidazole-1-carboselenoate (3h) White solid (25.5 mg, 68\%). Mp: 229.5-230.9 ${ }^{\circ} \mathrm{C} .{ }^{1} \mathbf{H}$ NMR (400 MHz, DMSO- $\left.d_{6}\right) \delta 11.47$ (s, 1H), 7.75 (s, 1H), $7.26(\mathrm{~d}, J=7.7 \mathrm{~Hz}, 2 \mathrm{H}), 7.09$ (d, $J=7.8 \mathrm{~Hz}, 2 \mathrm{H}), 6.85(\mathrm{~s}, 1 \mathrm{H}), 4.11(\mathrm{~s}, 2 \mathrm{H}), 2.25(\mathrm{~s}, 3 \mathrm{H}), 2.23$ (s, 3H), $2.22(\mathrm{~s}, 3 \mathrm{H}) .{ }^{13} \mathrm{C}$ NMR (100 MHz, DMSO-d $) \delta 164.7,152.3,135.8,135.7,133.0,129.8,129.0$, $128.9,126.4,124.2,114.7,110.5,27.9,20.7,19.6,19.5$. IR (neat): $v=3205,2916,1718,1690,1343$ $\mathrm{cm}^{-1}$. HRMS (ESI): calcd. for $\mathrm{C}_{18} \mathrm{H}_{18} \mathrm{~N}_{2} \mathrm{O}_{2} \mathrm{SeNa}[\mathrm{M}+\mathrm{Na}]^{+}:$397.0426, found: 397.0422 .<smiles>Cc1cc2[nH]c(=O)n(C(=O)[Se]Cc3cccc(Cl)c3)c2cc1C</smiles>

$S e$-(3-chlorobenzyl) 5,6-dimethyl-2-oxo-2,3-dihydro-1H-benzo[ $d]$ imidazole-1-carboselenoate (3i) White solid (27.9 mg, 71\%). Mp: 242.1-243.6 ${ }^{\circ} \mathrm{C} .{ }^{1} \mathbf{H}$ NMR (400 MHz, DMSO-d $\left.d_{6}\right) \delta 11.50$ (s, 1H), 7.73 (s, 1H), $7.44(\mathrm{~s}, 1 \mathrm{H}), 7.39-7.18(\mathrm{~m}, 4 \mathrm{H}), 6.84(\mathrm{~s}, 1 \mathrm{H}), 4.13(\mathrm{~s}, 2 \mathrm{H}), 2.21(\mathrm{~d}, J=4.5 \mathrm{~Hz}, 6 \mathrm{H}) .{ }^{13} \mathbf{C}$ NMR $\left(100 \mathrm{MHz}, \mathrm{DMSO}-d_{6}\right) \delta 164.5,152.4,142.3,133.1,132.9,130.3,129.9,128.8,127.8,126.6,126.5$, 124.2, 114.8, 110.6, 27.4, 19.60, 19.59. IR (neat): $v=3149,1916,1718,1682,1345 \mathrm{~cm}^{-1}$. HRMS (ESI): calcd. for $\mathrm{C}_{17} \mathrm{H}_{15} \mathrm{ClN}_{2} \mathrm{O}_{2} \mathrm{SeNa}[\mathrm{M}+\mathrm{Na}]^{+}: 416.9879$, found: 416.9889 .<smiles>CCOC(=O)CCC[Se]C(=O)n1c(=O)[nH]c2cc(C)c(C)cc21</smiles>

ethyl 4-((5,6-dimethyl-2-oxo-2,3-dihydro-1H-benzo[d] imidazole-1-carbonyl)selanyl)butanoate (3j) White solid (25.8 mg, 67\%). Mp: $135.9-137.4{ }^{\circ} \mathrm{C} .{ }^{1} \mathbf{H}$ NMR (400 MHz, DMSO-d $) \delta 11.45$ (s, 1H), 7.72 $(\mathrm{s}, 1 \mathrm{H}), 6.84(\mathrm{~s}, 1 \mathrm{H}), 4.06(\mathrm{q}, J=7.1 \mathrm{~Hz}, 2 \mathrm{H}), 2.88(\mathrm{t}, J=7.3 \mathrm{~Hz}, 2 \mathrm{H}), 2.40(\mathrm{t}, J=7.4 \mathrm{~Hz}, 2 \mathrm{H}), 2.21(\mathrm{~s}$, 6H), $1.94(\mathrm{p}, J=7.5 \mathrm{~Hz}, 2 \mathrm{H}), 1.18(\mathrm{t}, J=7.2 \mathrm{~Hz}, 3 \mathrm{H}) .{ }^{13} \mathbf{C}$ NMR (100 MHz, DMSO-d $) \delta 172.3,164.4$, $152.3,132.9,129.7,126.3,124.3,114.8,110.4,59.8,33.6,25.1,23.7,19.55,19.51,14.1$. IR (neat): $v=$ 
3157, 2971, 1712, 1683, $1345 \mathrm{~cm}^{-1}$. HRMS (ESI): calcd. for $\mathrm{C}_{16} \mathrm{H}_{20} \mathrm{~N}_{2} \mathrm{O}_{4} \mathrm{SeNa}[\mathrm{M}+\mathrm{Na}]^{+}:$407.0481, found: 407.0479 .

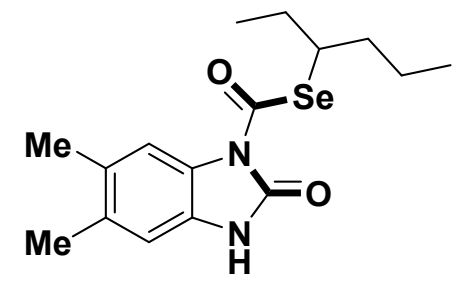

Se-(hexan-3-yl) 5,6-dimethyl-2-oxo-2,3-dihydro-1H-benzo[d]imidazole-1-carboselenoate (3k)

White solid (25.9 mg, 73\%). Mp: $116.1-117.5^{\circ} \mathrm{C} .{ }^{1} \mathbf{H}$ NMR (400 MHz, DMSO-d $)_{6} \delta 11.43$ (s, $\left.1 \mathrm{H}\right), 7.73$ $(\mathrm{s}, 1 \mathrm{H}), 6.83(\mathrm{~s}, 1 \mathrm{H}), 3.53(\mathrm{p}, J=6.7 \mathrm{~Hz}, 1 \mathrm{H}), 2.21(\mathrm{~s}, 7 \mathrm{H}), 1.70(\mathrm{dq}, J=26.1,7.1 \mathrm{~Hz}, 4 \mathrm{H}), 1.41(\mathrm{dq}, J$ $=22.0,7.0 \mathrm{~Hz}, 2 \mathrm{H}), 0.96(\mathrm{t}, J=7.3 \mathrm{~Hz}, 3 \mathrm{H}), 0.89(\mathrm{t}, J=7.4 \mathrm{~Hz}, 3 \mathrm{H}) .{ }^{13} \mathbf{C}$ NMR $\left(100 \mathrm{MHz}, \mathrm{DMSO}-d_{6}\right)$ $\delta 164.9,152.2,132.8,129.6,126.3,124.4,114.9,110.4,44.1,36.1,27.5,20.5,19.53,19.51,13.8,12.0$. IR (neat): $v=3152,2957,2923,1717,1669,1339 \mathrm{~cm}^{-1}$. HRMS (ESI): calcd. for $\mathrm{C}_{16} \mathrm{H}_{22} \mathrm{~N}_{2} \mathrm{O}_{2} \mathrm{SeNa}$ $[\mathrm{M}+\mathrm{Na}]^{+}:$377.0739, found: 377.0738 .<smiles>Cc1cc2[nH]c(=O)n(C(=O)C3CCCCC3)c2cc1C</smiles>

Se-cyclohexyl 5,6-dimethyl-2-oxo-2,3-dihydro-1 $H$-benzo $[d]$ imidazole-1-carboselenoate (3) Off-white solid (22.6 mg, 64\%). Mp: 224.8-226.0 ${ }^{\circ} \mathrm{C} .{ }^{1} \mathbf{H}$ NMR (400 MHz, DMSO-d $)_{6} \delta 11.43(\mathrm{~s}, 1 \mathrm{H})$, $7.75(\mathrm{~s}, 1 \mathrm{H}), 6.84(\mathrm{~s}, 1 \mathrm{H}), 3.57(\mathrm{tt}, J=10.3,3.2 \mathrm{~Hz}, 1 \mathrm{H}), 3.35(\mathrm{~s}, 8 \mathrm{H}), 2.22(\mathrm{~s}, 5 \mathrm{H}), 2.06-1.96(\mathrm{~m}, 2 \mathrm{H})$, $1.67(\mathrm{dt}, J=12.9,4.2 \mathrm{~Hz}, 2 \mathrm{H}), 1.58(\mathrm{~h}, J=9.9 \mathrm{~Hz}, 3 \mathrm{H}), 1.49-1.35(\mathrm{~m}, 2 \mathrm{H}), 1.32(\mathrm{t}, J=11.3 \mathrm{~Hz}, 1 \mathrm{H})$. ${ }^{13}$ C NMR (100 MHz, DMSO- $\left.d_{6}\right) \delta 164.6,152.2,132.9,129.7,126.4,124.4,114.9,110.4,40.0,32.9$, 26.4, 25.3, 19.6, 19.5. IR (neat): $v=3143,2929,2847,1713,1667,1331 \mathrm{~cm}^{-1}$. HRMS (ESI): calcd. for $\mathrm{C}_{16} \mathrm{H}_{20} \mathrm{~N}_{2} \mathrm{O}_{2} \mathrm{SeNa}[\mathrm{M}+\mathrm{Na}]^{+}: 375.0582$, found: 375.0590 . 


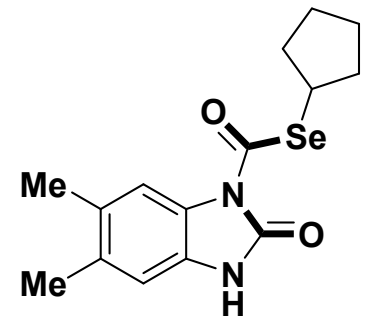

Se-cyclopentyl 5,6-dimethyl-2-oxo-2,3-dihydro-1 $H$-benzo[d]imidazole-1-carboselenoate $(3 \mathrm{~m})$

Pale yellow solid (24.4 mg, 72\%). Mp: 203.2-204. ${ }^{\circ} \mathrm{C} .{ }^{1} \mathbf{H}$ NMR (400 MHz, DMSO-d $) \delta 11.42(\mathrm{~s}, 1 \mathrm{H})$, $7.73(\mathrm{~s}, 1 \mathrm{H}), 6.83(\mathrm{~s}, 1 \mathrm{H}), 3.72(\mathrm{p}, J=7.5 \mathrm{~Hz}, 1 \mathrm{H}), 2.21(\mathrm{~s}, 6 \mathrm{H}), 2.17-2.09(\mathrm{~m}, 2 \mathrm{H}), 1.72-1.55(\mathrm{~m}$, 6H). ${ }^{13}$ C NMR (100 MHz, DMSO-d $\left.{ }_{6}\right) \delta 165.2,152.3,132.8,129.6,126.3,124.3,114.8,110.4,38.8$, 32.8, 24.7, 19.6, 19.5. IR (neat): $v=3132,2945,2860,1714,1675,1339 \mathrm{~cm}^{-1}$. HRMS (ESI): calcd. for $\mathrm{C}_{15} \mathrm{H}_{18} \mathrm{~N}_{2} \mathrm{O}_{2} \mathrm{SeNa}[\mathrm{M}+\mathrm{Na}]^{+}: 361.0426$, found: 361.0420 .

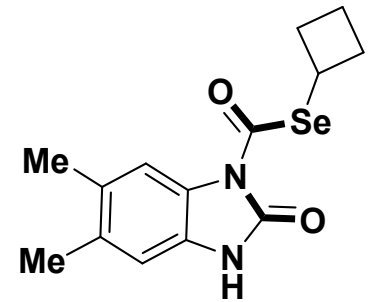

Se-cyclobutyl 5,6-dimethyl-2-oxo-2,3-dihydro-1H-benzo[d]imidazole-1-carboselenoate (3n)

Pale yellow solid (24.3 mg, 75\%). Mp: 208.8-210.2 ${ }^{\circ} \mathrm{C} .{ }^{1} \mathbf{H}$ NMR (400 MHz, DMSO- $\left.d_{6}\right) \delta 11.41(\mathrm{~s}, 1 \mathrm{H})$, $7.69(\mathrm{~s}, 1 \mathrm{H}), 6.81(\mathrm{~s}, 1 \mathrm{H}), 4.15(\mathrm{p}, J=8.0 \mathrm{~Hz}, 1 \mathrm{H}), 2.47-2.39(\mathrm{~m}, 2 \mathrm{H}), 2.19(\mathrm{~s}, 8 \mathrm{H}), 2.07-1.96(\mathrm{~m}$, 2H). ${ }^{13}$ C NMR (100 MHz, DMSO-d $\left.d_{6}\right) \delta 164.6,152.2,132.9,129.7,126.3,124.3,114.8,110.4,33.2$, 29.9, 20.3, 19.6, 19.5. IR (neat): $v=3160,2967,2934,2855,1717,1673,1481,1339 \mathrm{~cm}^{-1}$. HRMS (ESI): calcd. for $\mathrm{C}_{14} \mathrm{H}_{16} \mathrm{~N}_{2} \mathrm{O}_{2} \mathrm{SeNa}[\mathrm{M}+\mathrm{Na}]^{+}:$347.0269, found: 347.0258 .<smiles>Cc1cc2[nH]c(=O)n(C(=O)NCc3ccccc3)c2cc1C</smiles>

$N$-benzyl-5,6-dimethyl-2-oxo-2,3-dihydro-1 $H$-benzo $[d]$ imidazole-1-carboxamide (4)

Off-white solid (22.0 mg, 75\%). Mp: 248.4-249.2 ${ }^{\circ} \mathrm{C} .{ }^{1} \mathbf{H}$ NMR (400 MHz, DMSO- $\left.d_{6}\right) \delta 11.28(\mathrm{~s}, 1 \mathrm{H})$, $9.14(\mathrm{t}, J=6.0 \mathrm{~Hz}, 1 \mathrm{H}), 7.79(\mathrm{~s}, 1 \mathrm{H}), 7.39-7.32(\mathrm{~m}, 4 \mathrm{H}), 7.29-7.25(\mathrm{~m}, 1 \mathrm{H}), 6.86(\mathrm{~s}, 1 \mathrm{H}), 4.52(\mathrm{~d}, J$ $=5.9 \mathrm{~Hz}, 2 \mathrm{H}), 2.23(\mathrm{~s}, 6 \mathrm{H}) .{ }^{13} \mathbf{C}$ NMR $\left(100 \mathrm{MHz}, \mathrm{DMSO}-d_{6}\right) \delta 153.6,151.4,138.9,131.4,129.4,128.4$, 
127.4, 127.1, 125.9, 125.3, 115.2, 110.2, 42.8, 19.7, 19.4. IR (neat): $v=3157,1748,1669,1557,1346$ $\mathrm{cm}^{-1}$. HRMS (ESI): calcd. for $\mathrm{C}_{17} \mathrm{H}_{18} \mathrm{~N}_{3} \mathrm{O}_{2}[\mathrm{M}+\mathrm{H}]^{+}:$296.1394, found: 296.1390 .

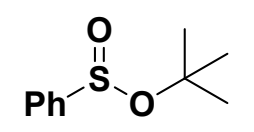

tert-butyl benzenesulfinate (5)

Pale yellow oil (33.7 mg, 34\%). ${ }^{1} \mathbf{H}$ NMR (400 MHz, $\left.\mathrm{CDCl}_{3}\right) \delta 7.72-7.63(\mathrm{~m}, 2 \mathrm{H}), 7.56-7.45(\mathrm{~m}, 3 \mathrm{H})$, 1.56 (s, 9H). ${ }^{13} \mathbf{C}$ NMR $\left(100 \mathrm{MHz}, \mathrm{CDCl}_{3}\right) \delta 146.7,131.6,129.0,125.0,82.9,30.0$. Spectroscopic data are in accordance with those described in the literature. ${ }^{2}$ 
9. Copies of ${ }^{1} \mathrm{H}$ and ${ }^{13} \mathrm{C}$ NMR spectra for compounds<smiles>Cc1cc2[nH]c(=O)n(C(=O)[Se]CCOc3ccccc3)c2cc1C</smiles>
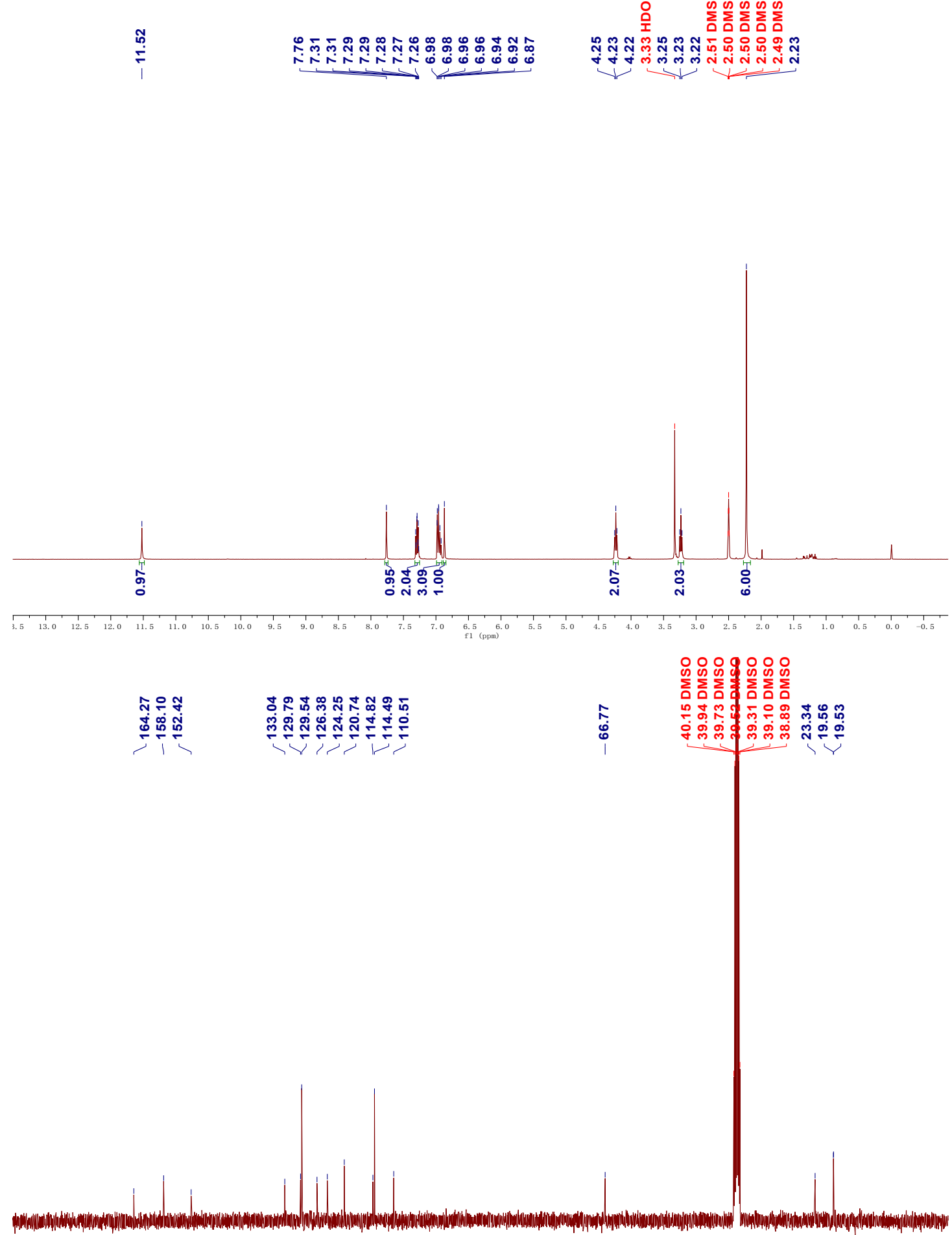

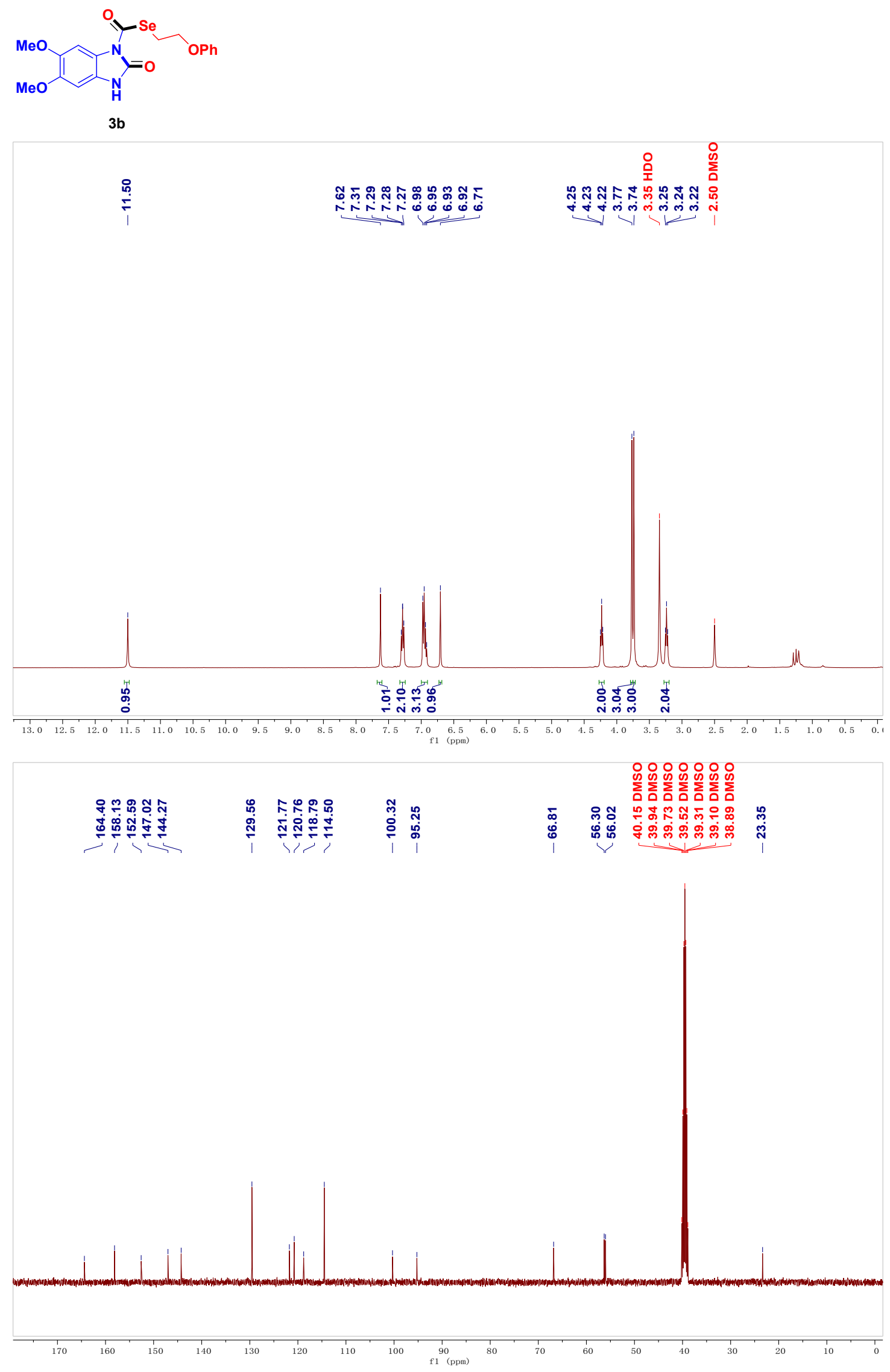


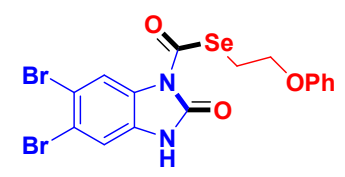

$3 c$

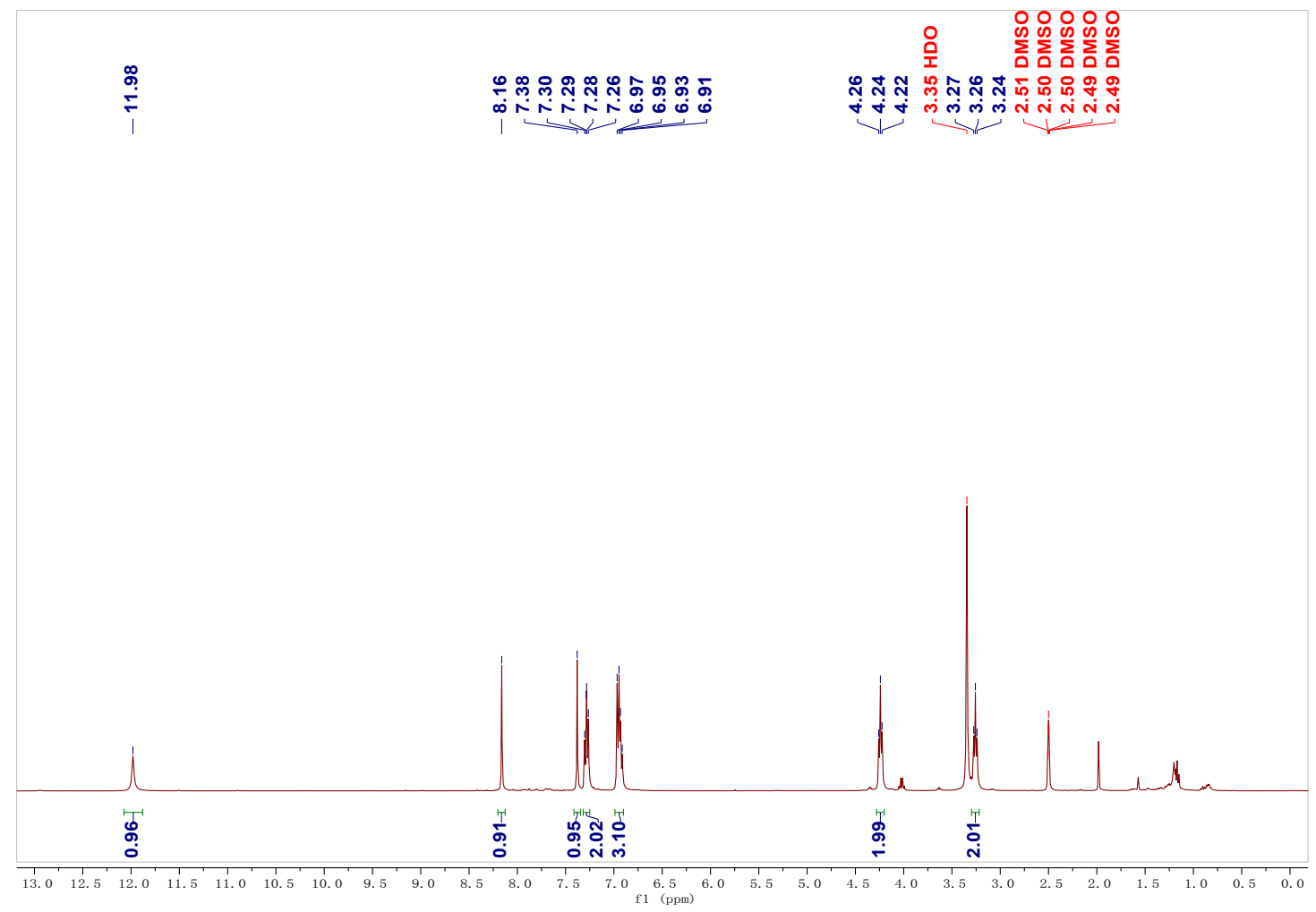

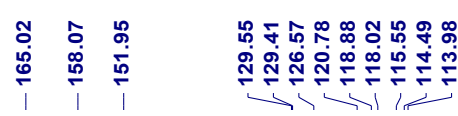

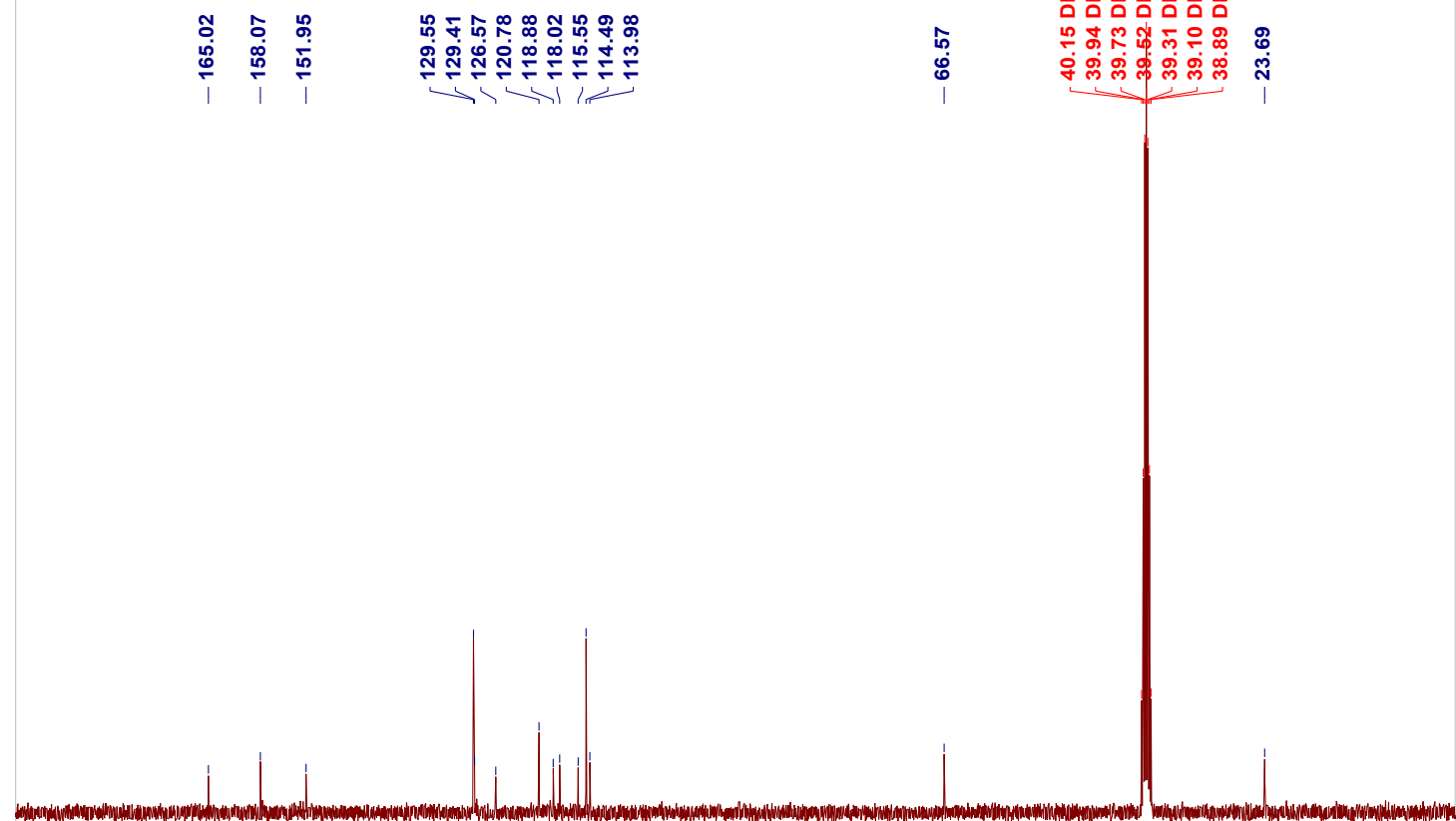




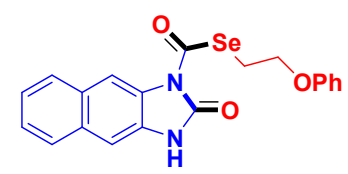

$3 d$

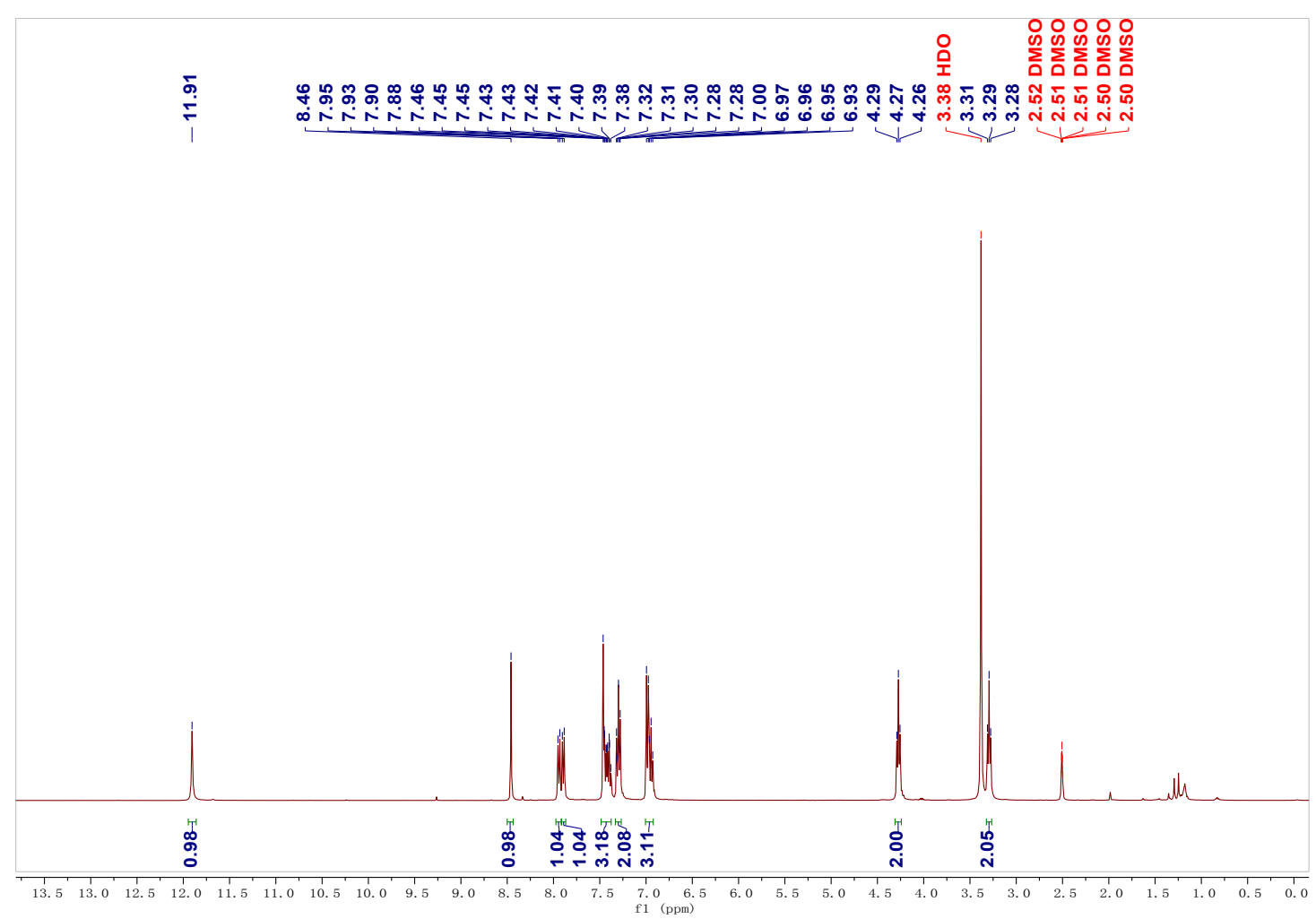

ริำ

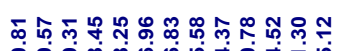

递总 뜰

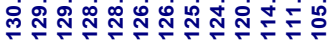

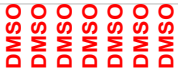

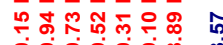

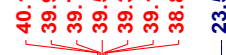

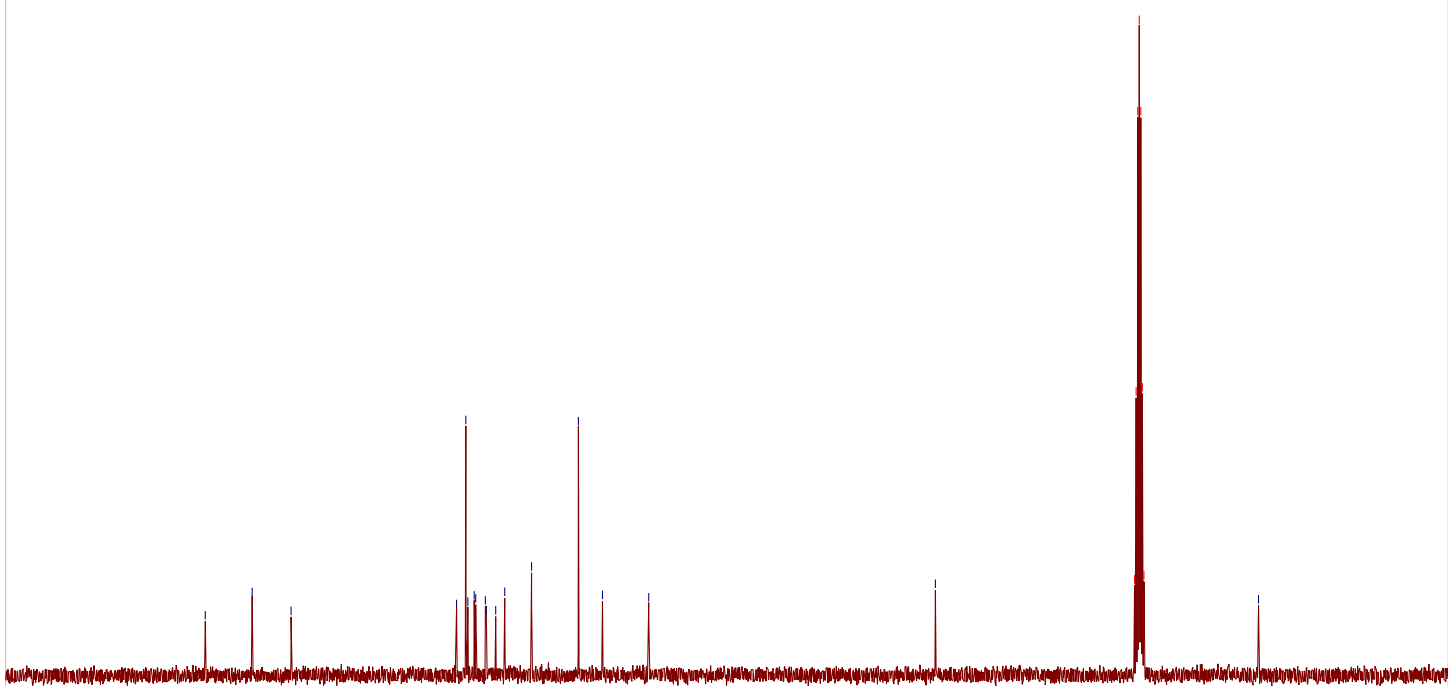

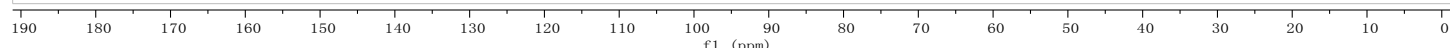




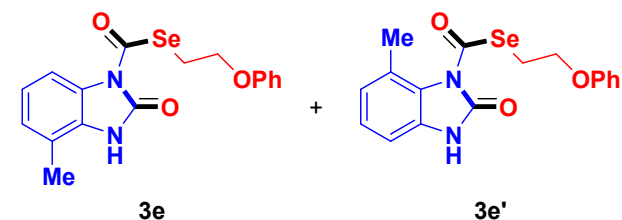

\section{ง
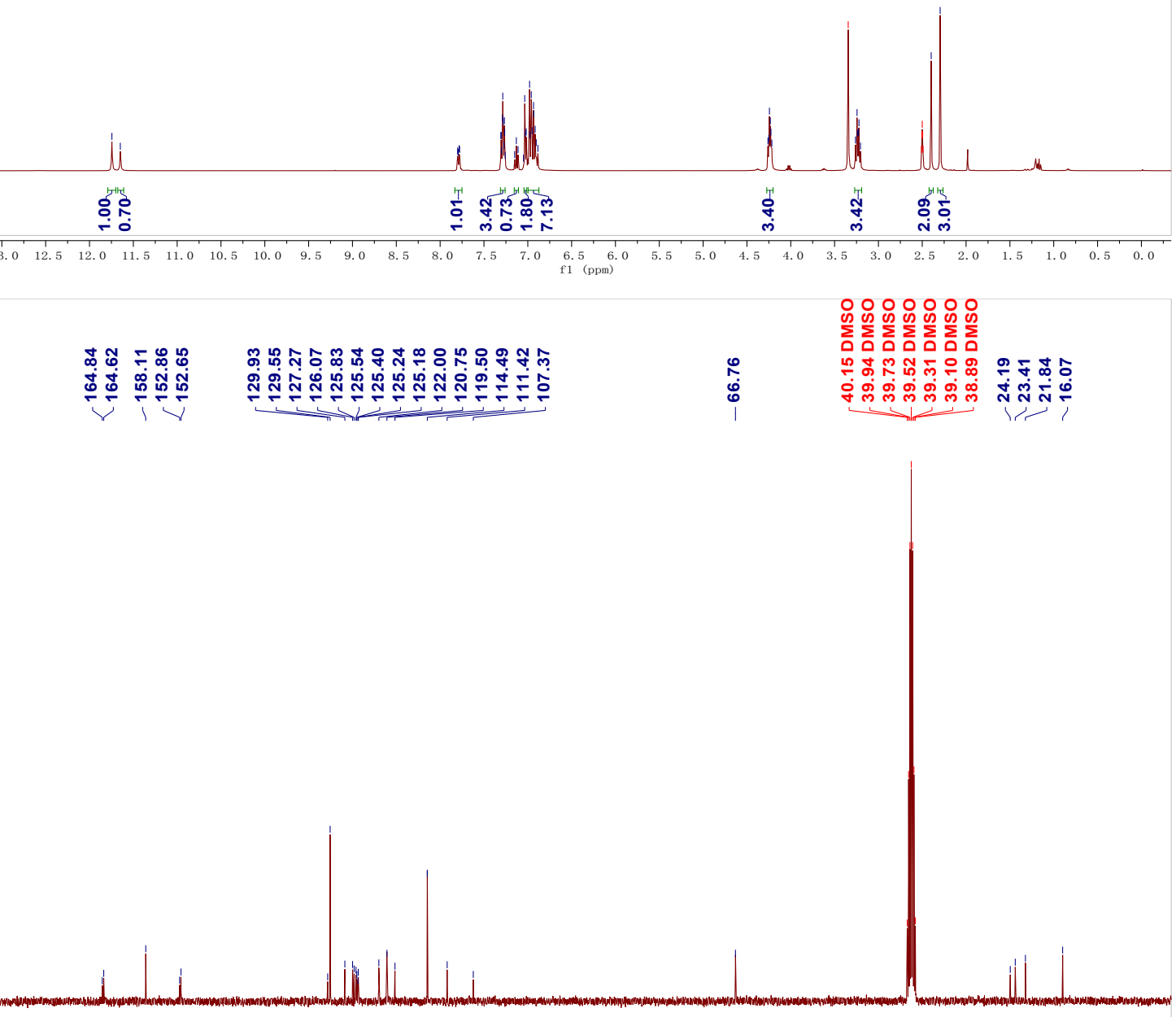

$\bar{T}_{180}$

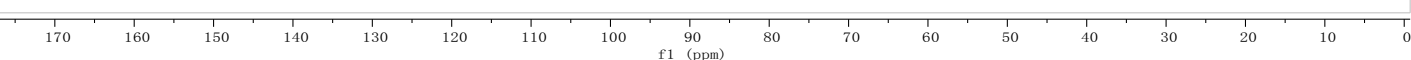




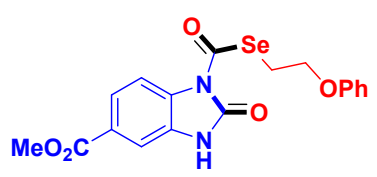

$3 f$
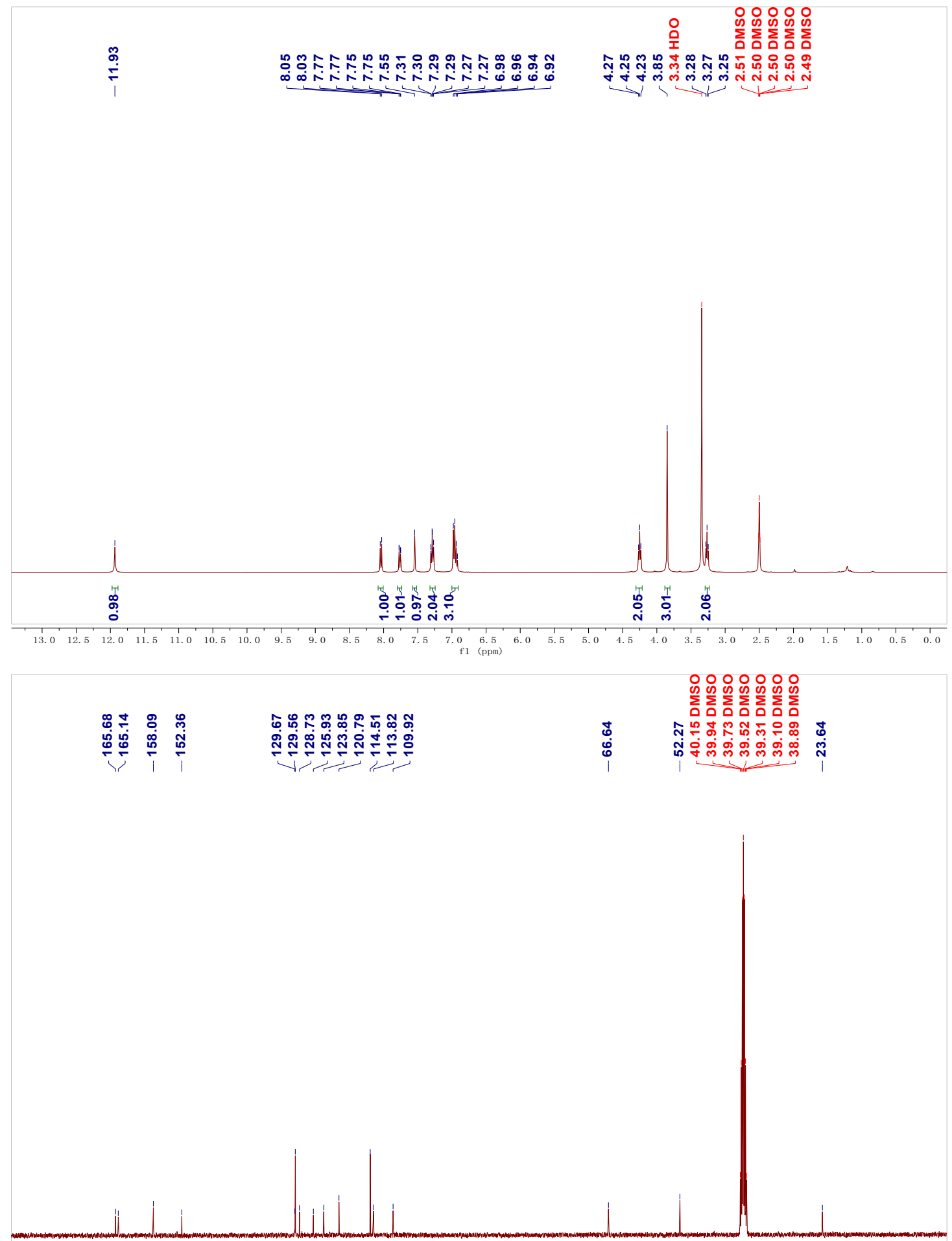

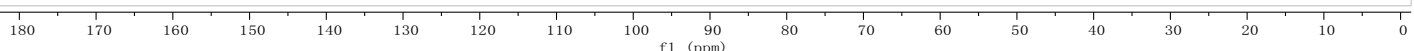


$\mathrm{MeO}_{2} \mathrm{C}$

3f'

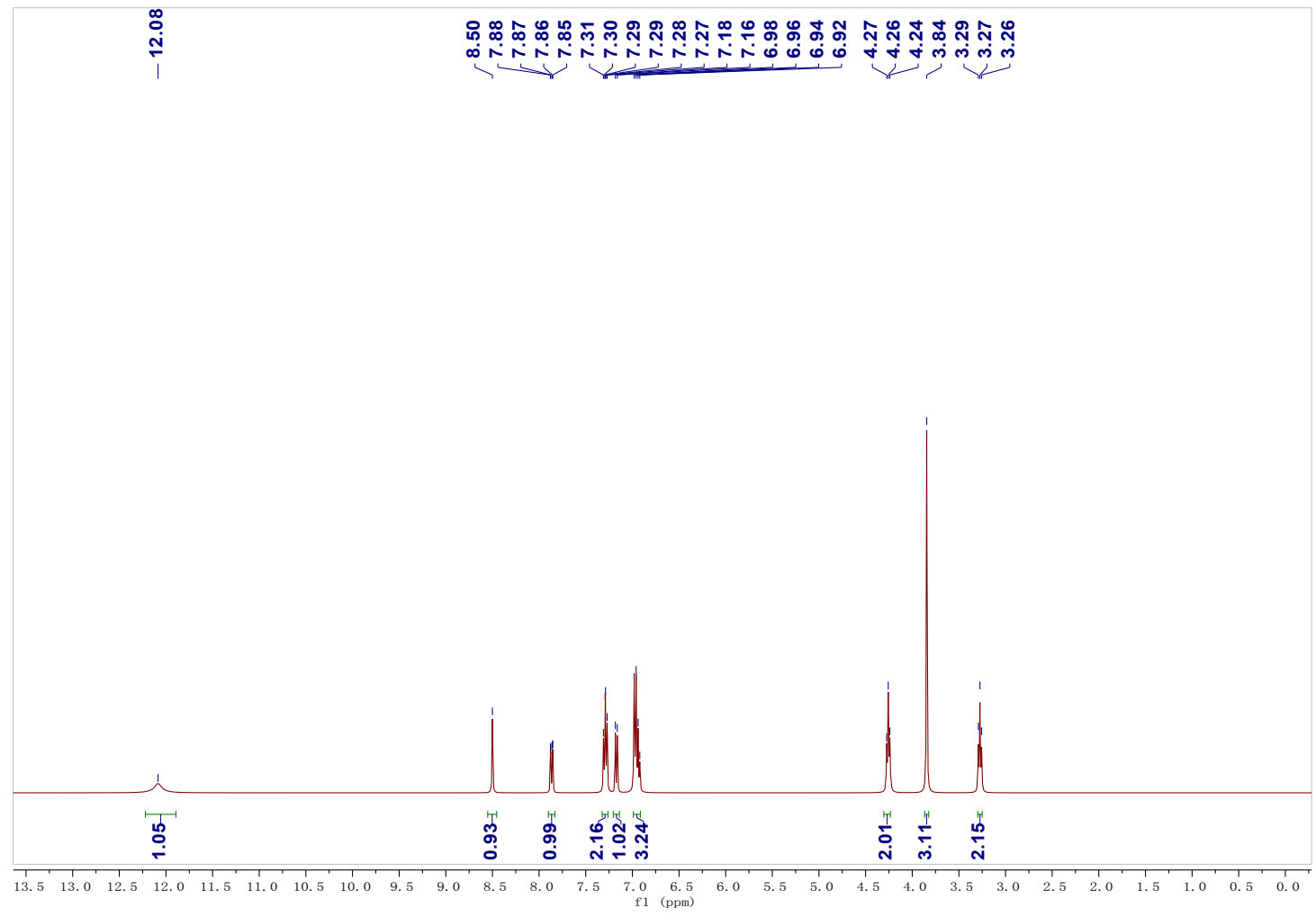

œ

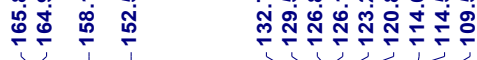

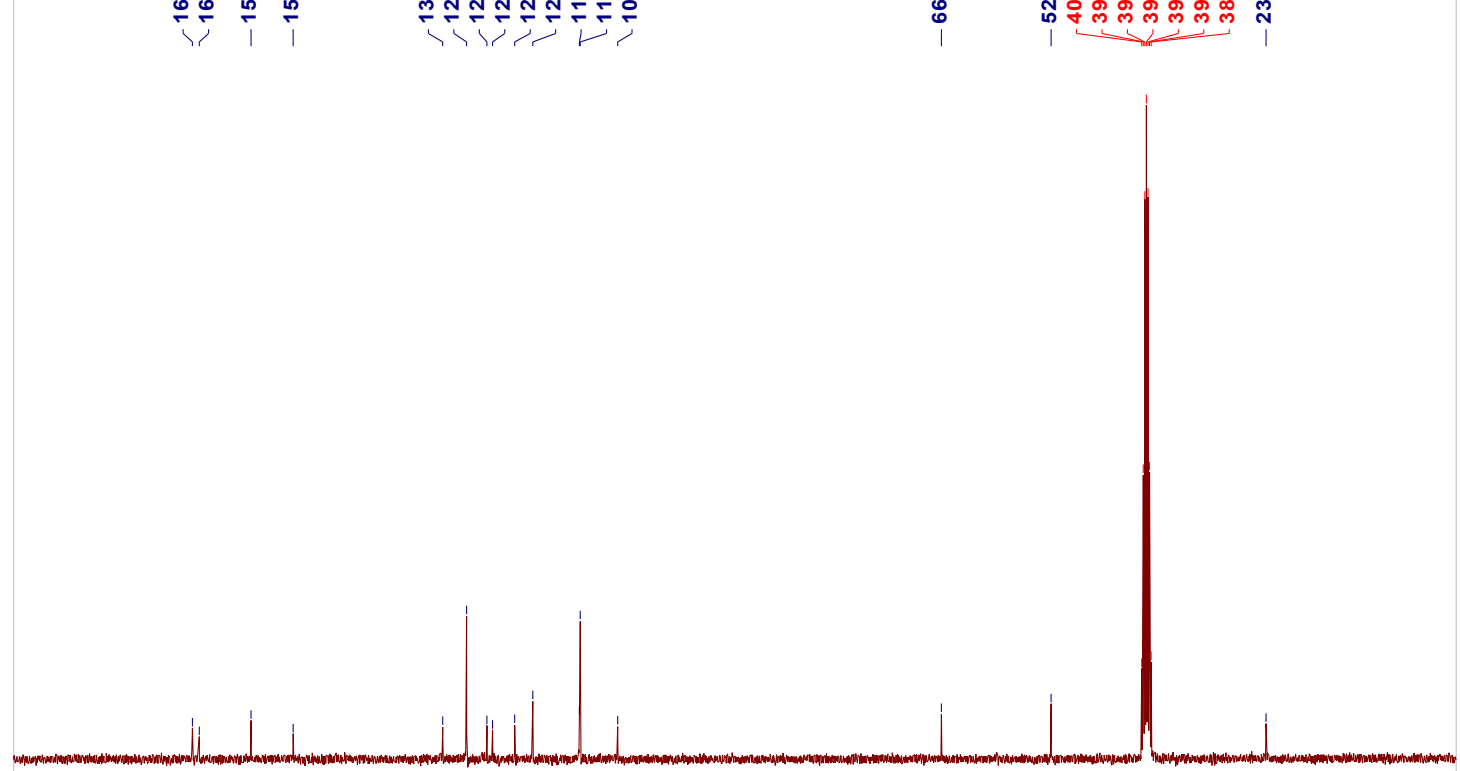

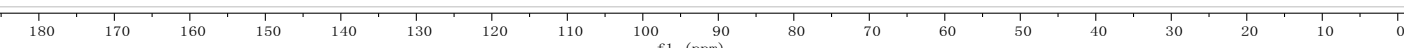




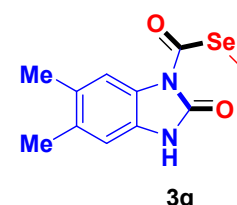

$3 \mathrm{~g}$

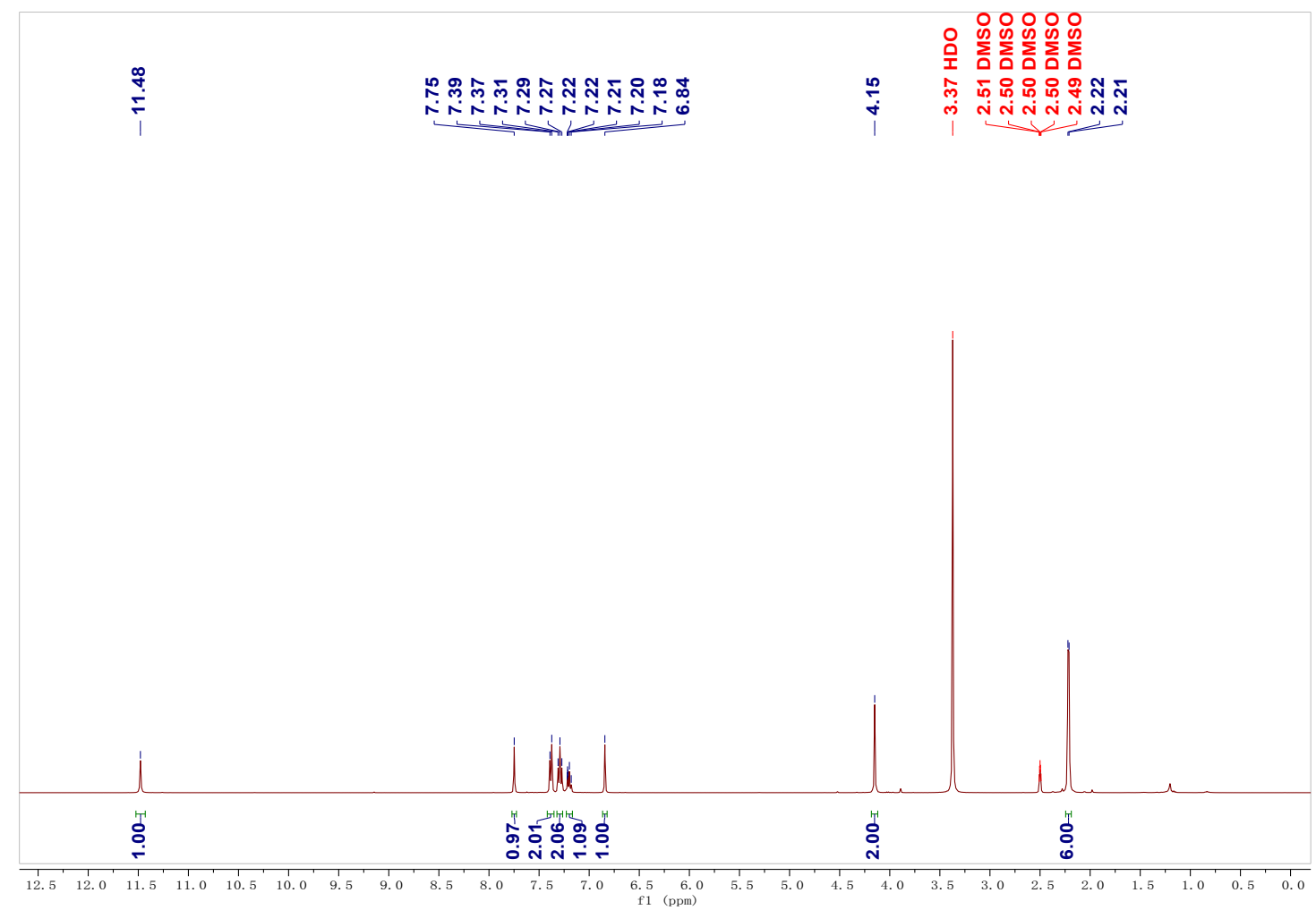

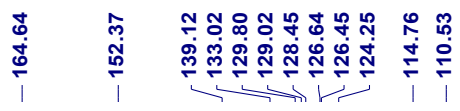

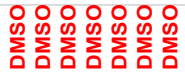

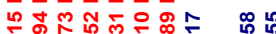

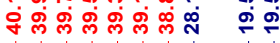
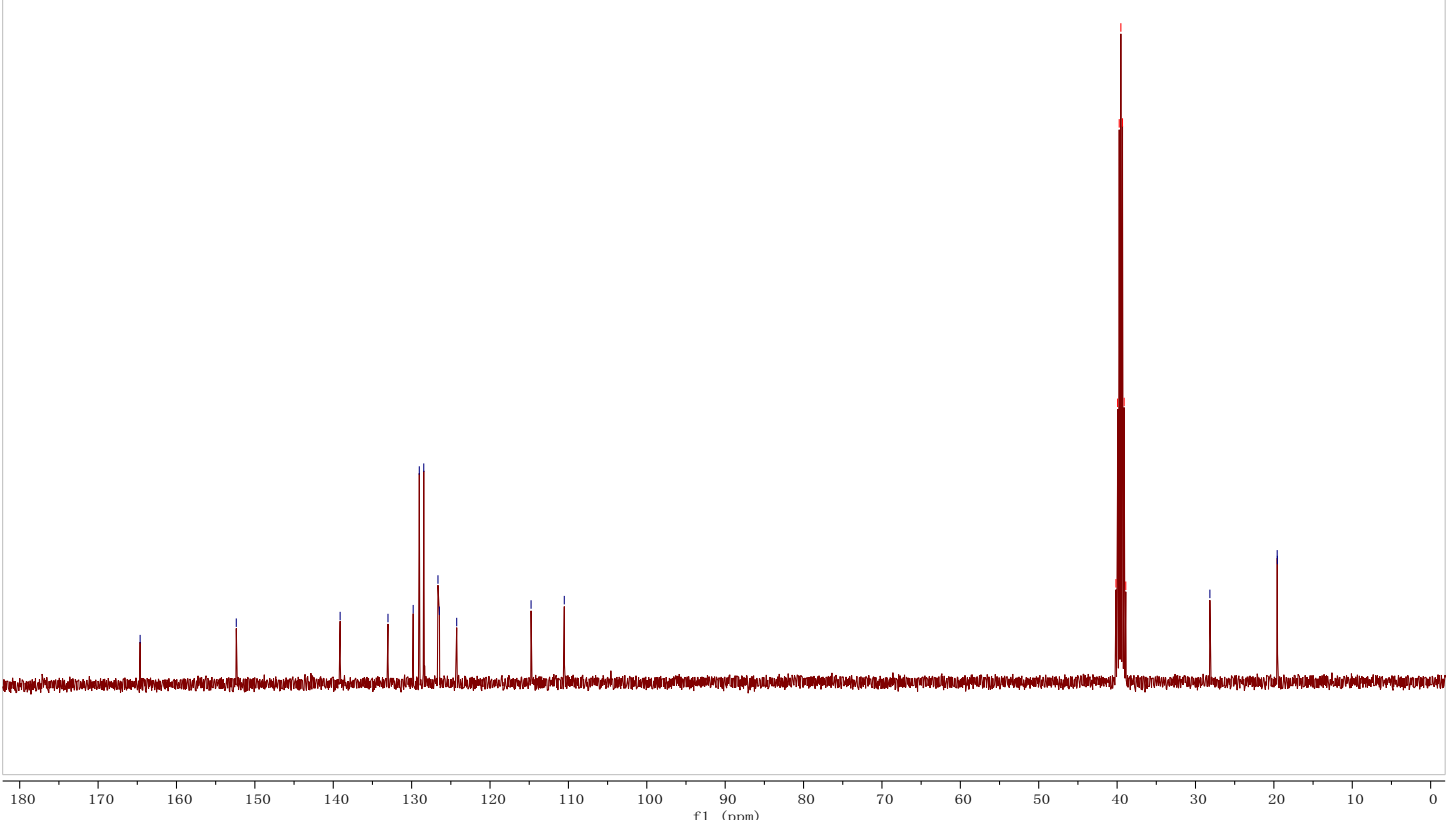


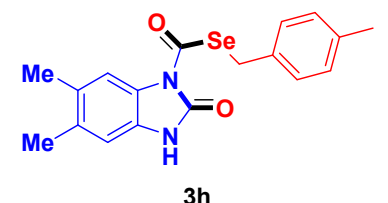

3h

$\stackrel{5}{\check{5}}$

ำง ำำำ

rivita

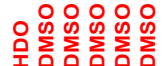

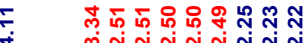
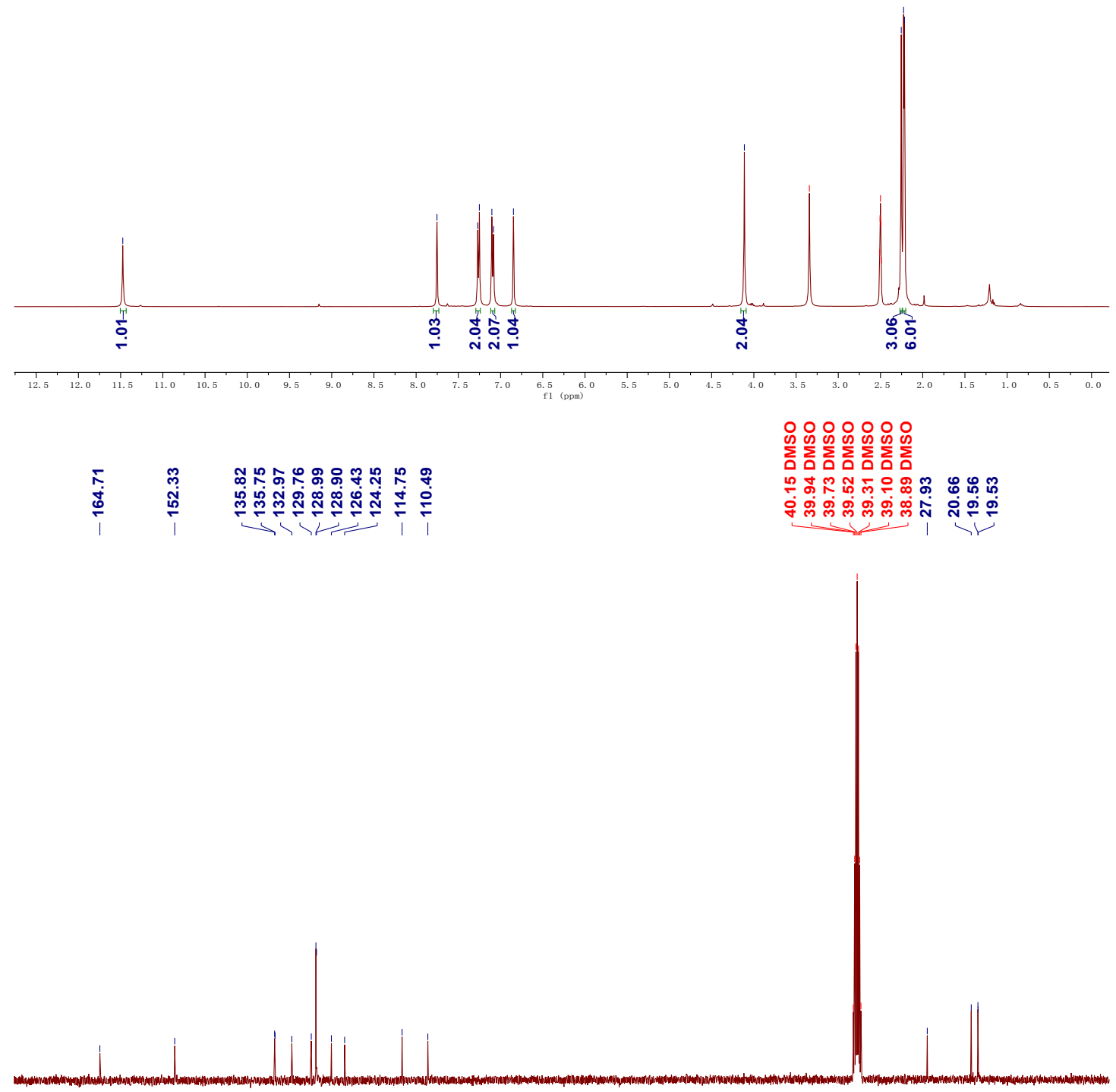


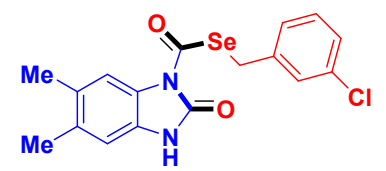

$3 \mathbf{i}$

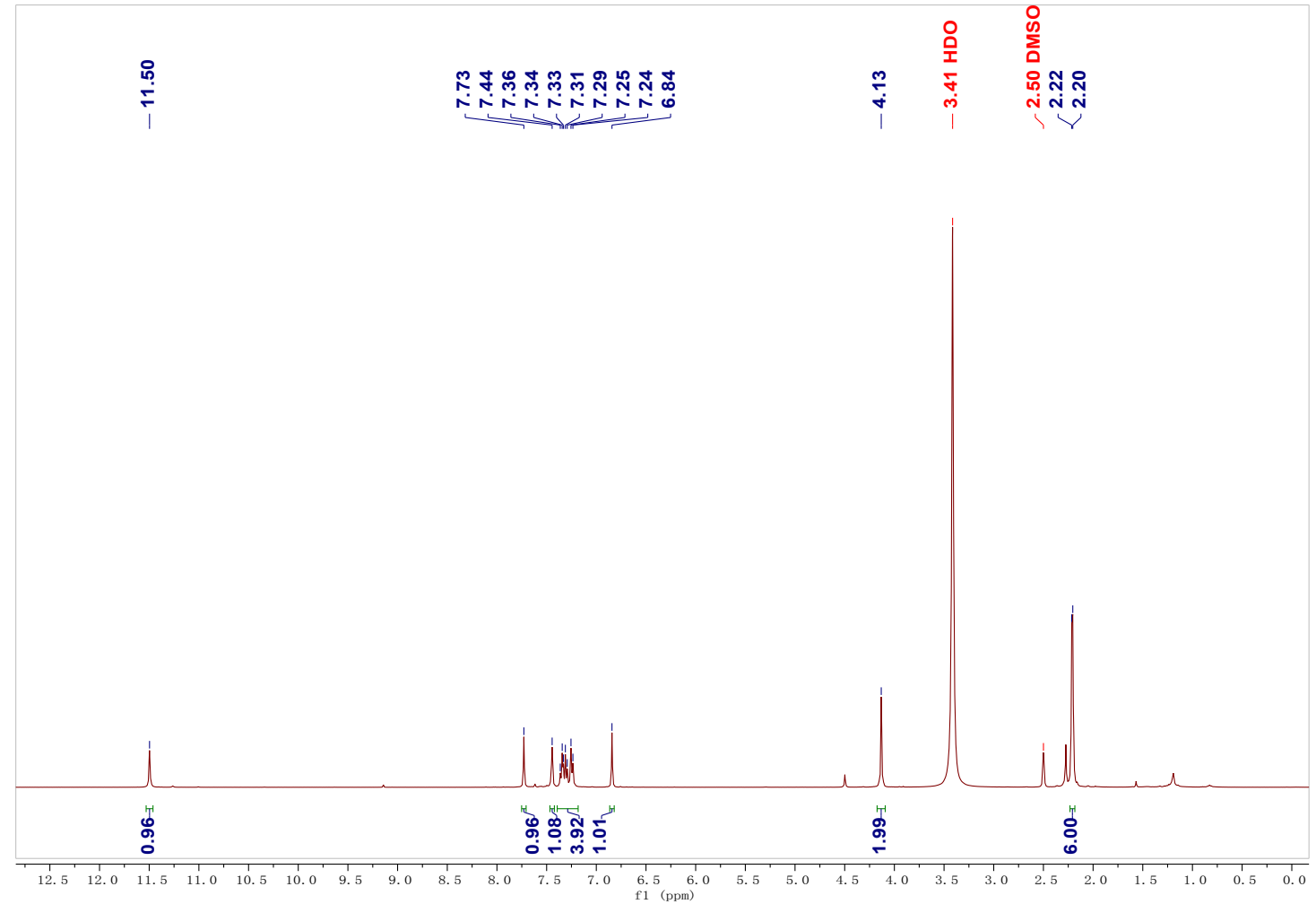

至

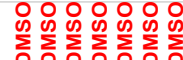

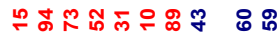

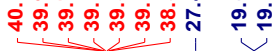

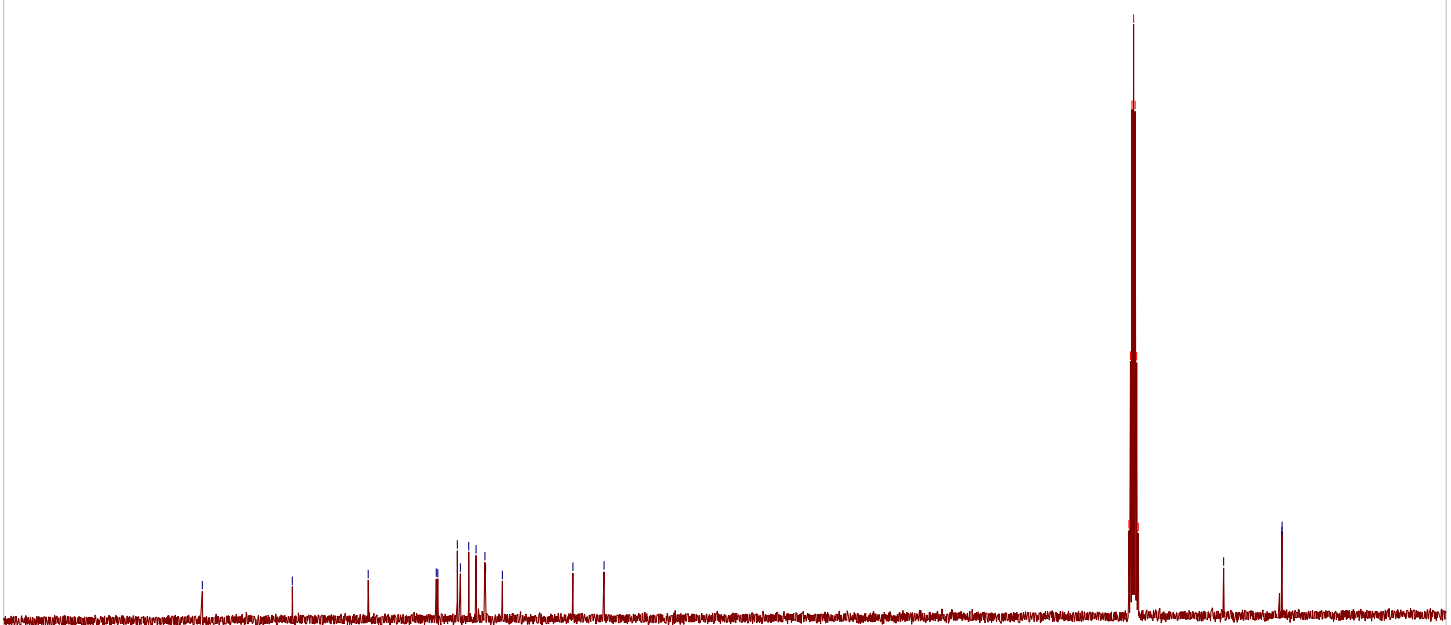

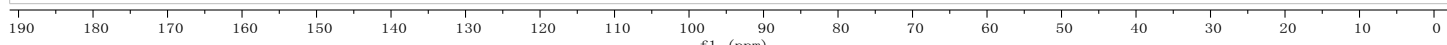


<smiles>CCOC(=O)CCCCC(=O)n1c(=O)[nH]c2cc(C)c(C)cc21</smiles>

3j

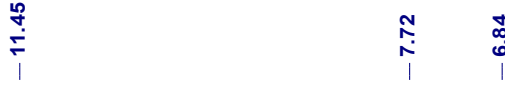

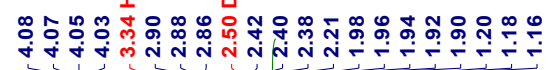
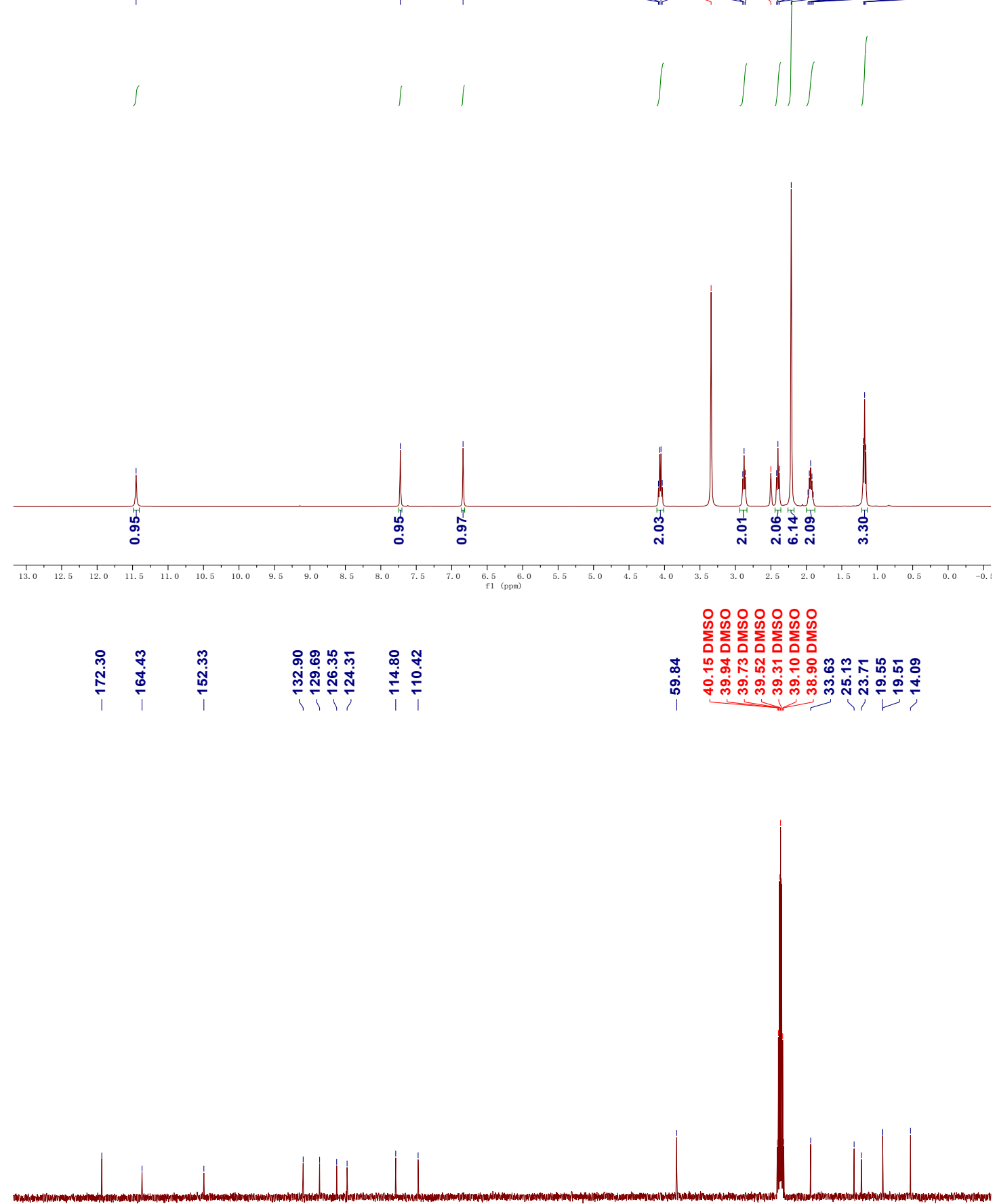
<smiles>CCCC(CC)C(=O)n1c(=O)[nH]c2cc(C)c(C)cc21</smiles>

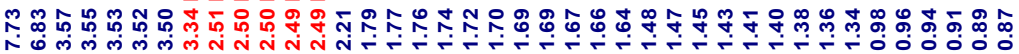

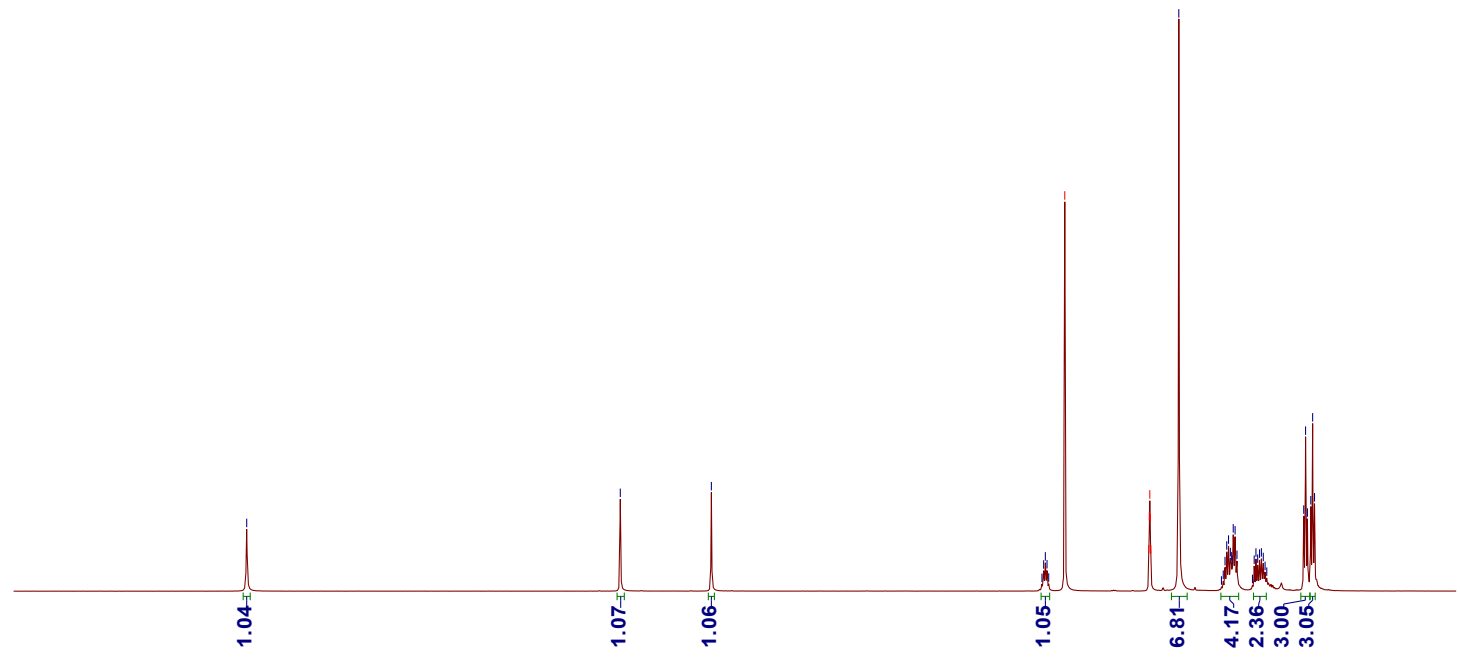

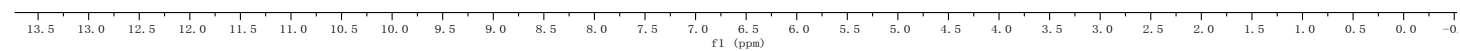

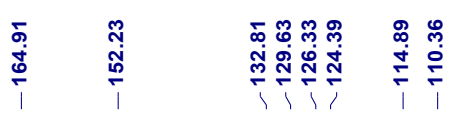

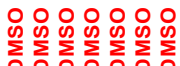

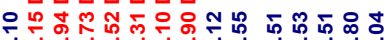

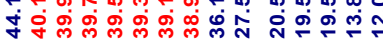
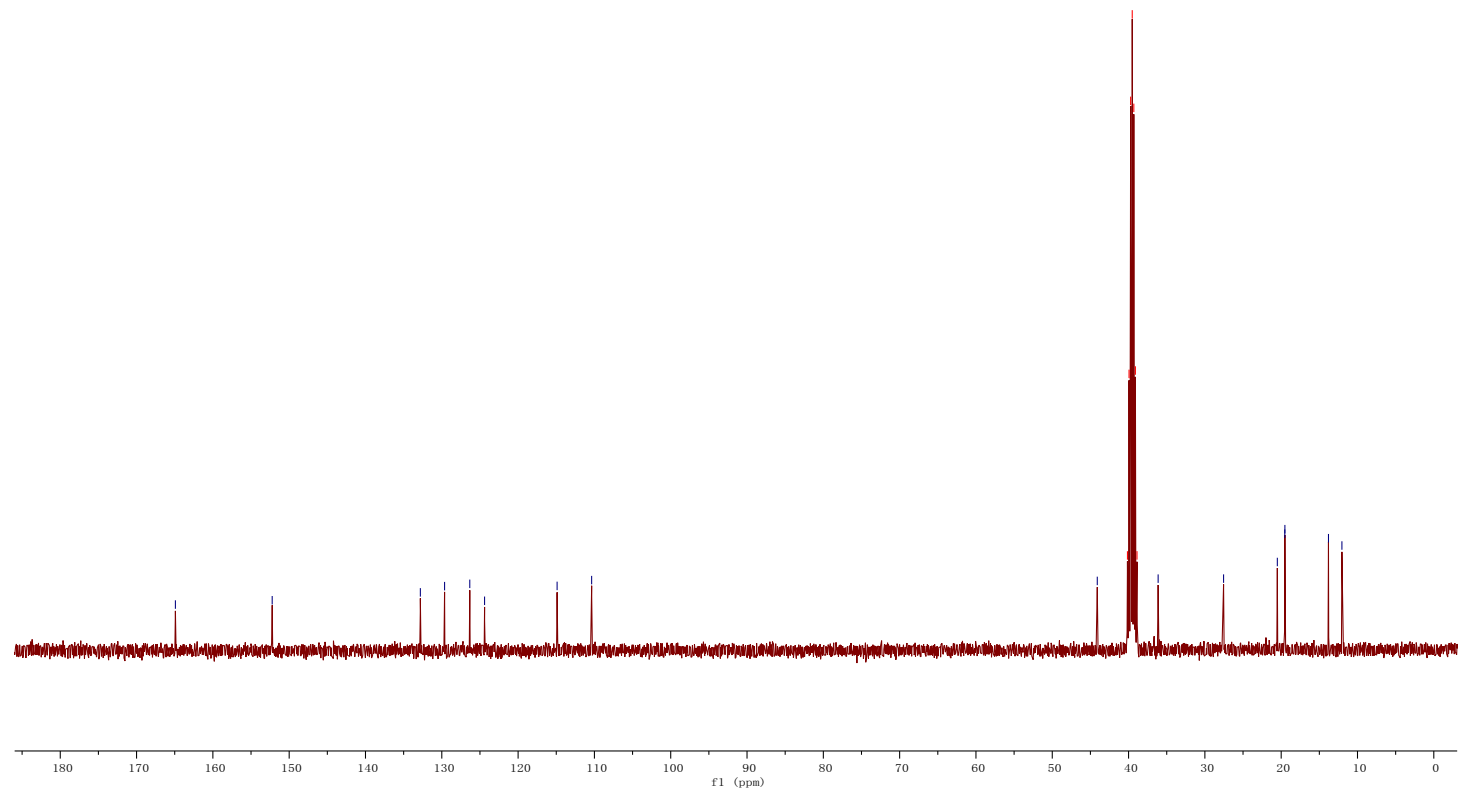


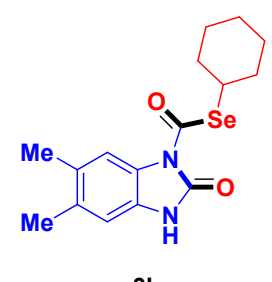

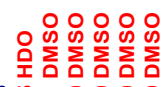

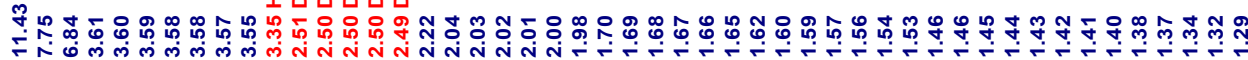

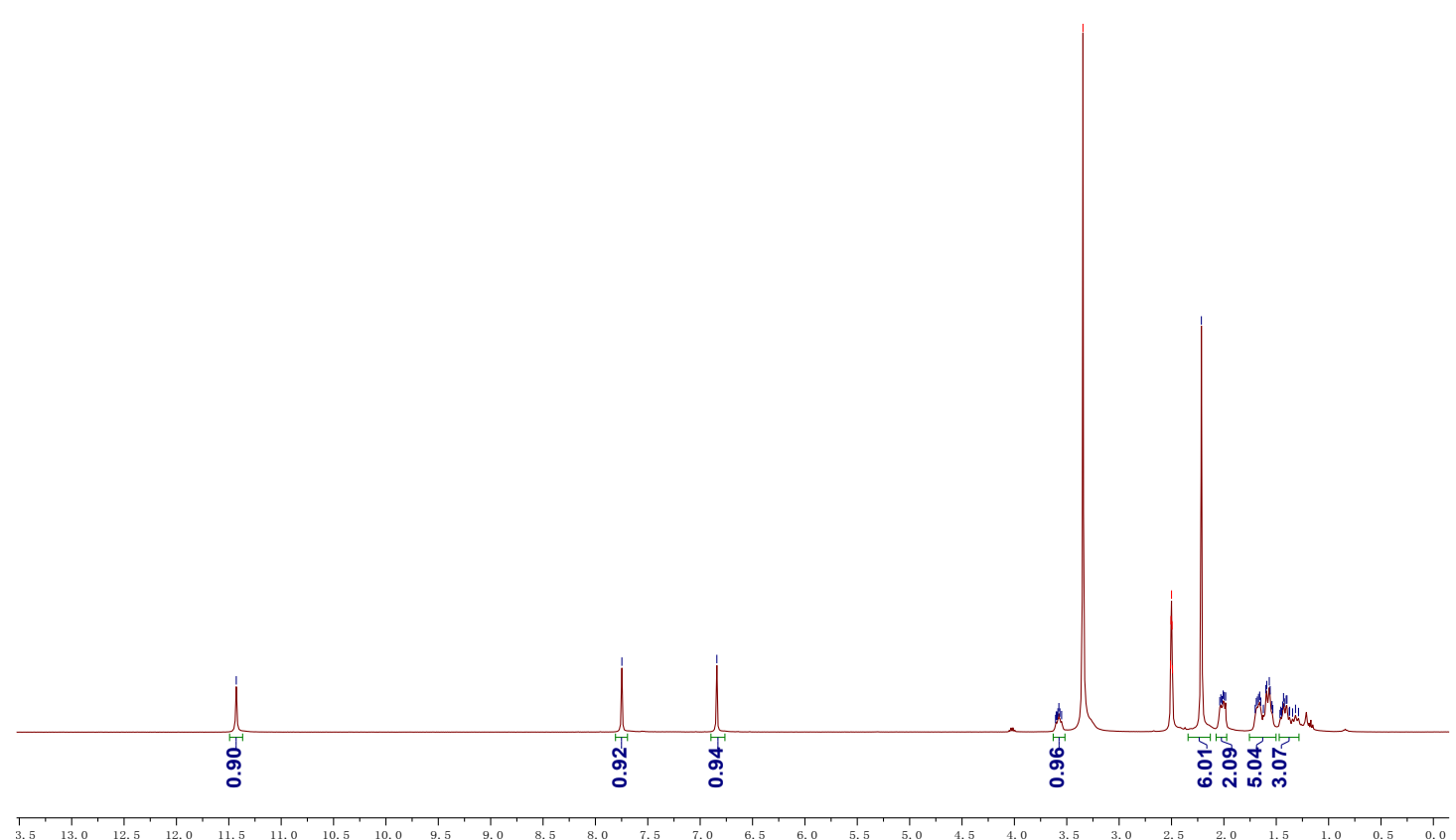

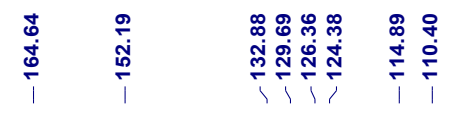

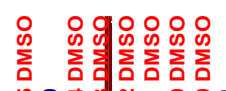

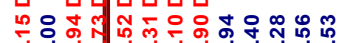

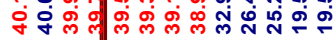


(c)

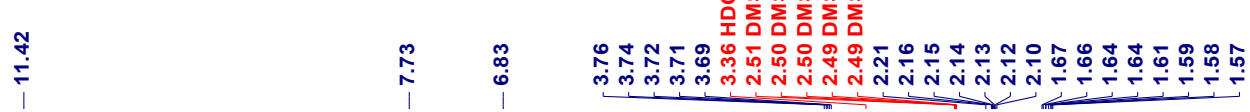
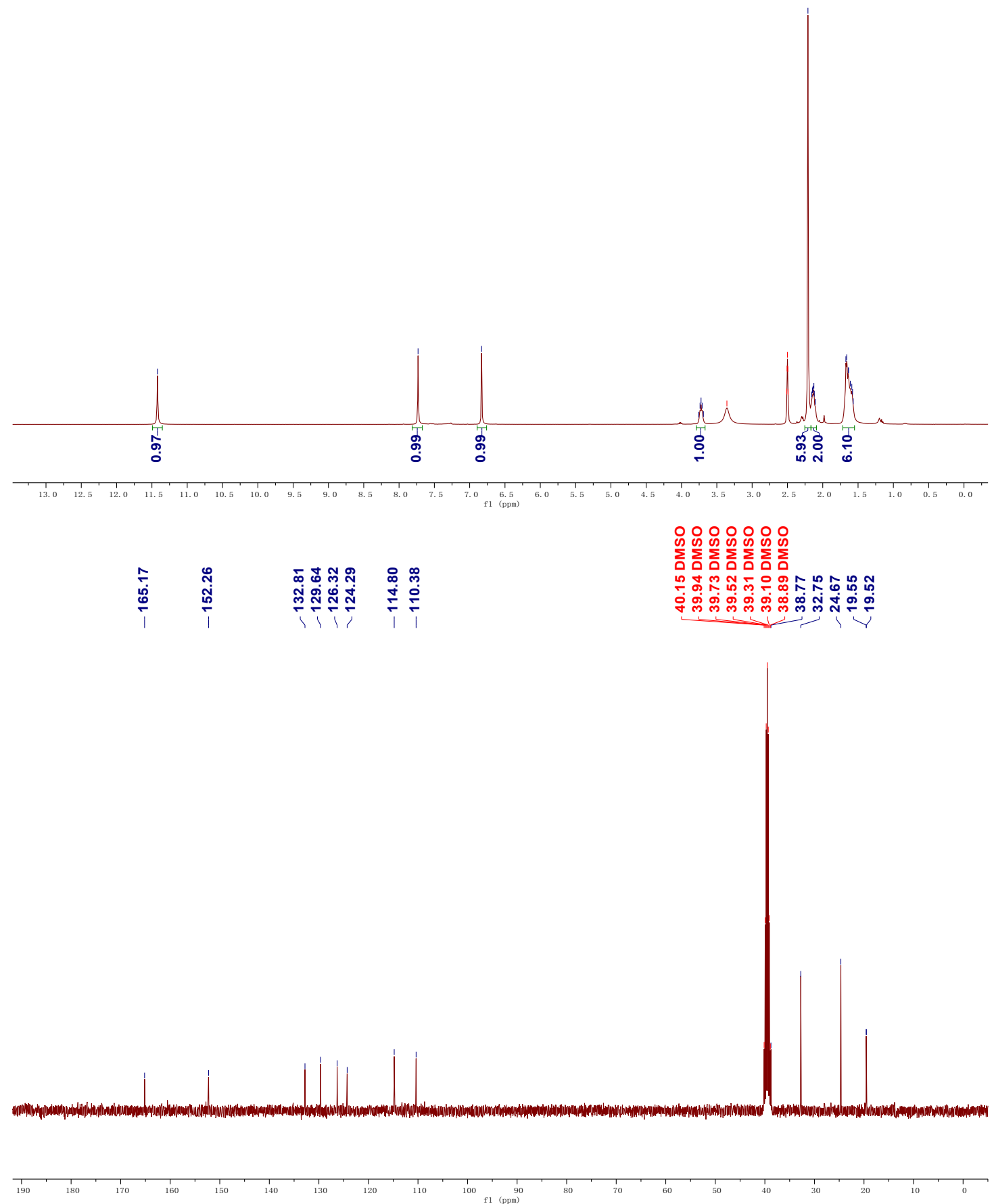
(Ne)

$3 n$

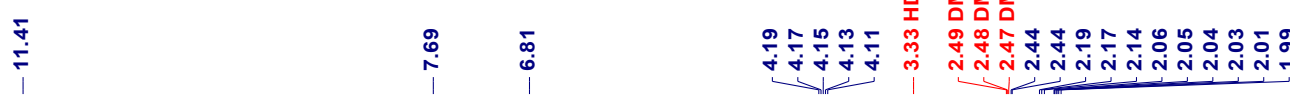
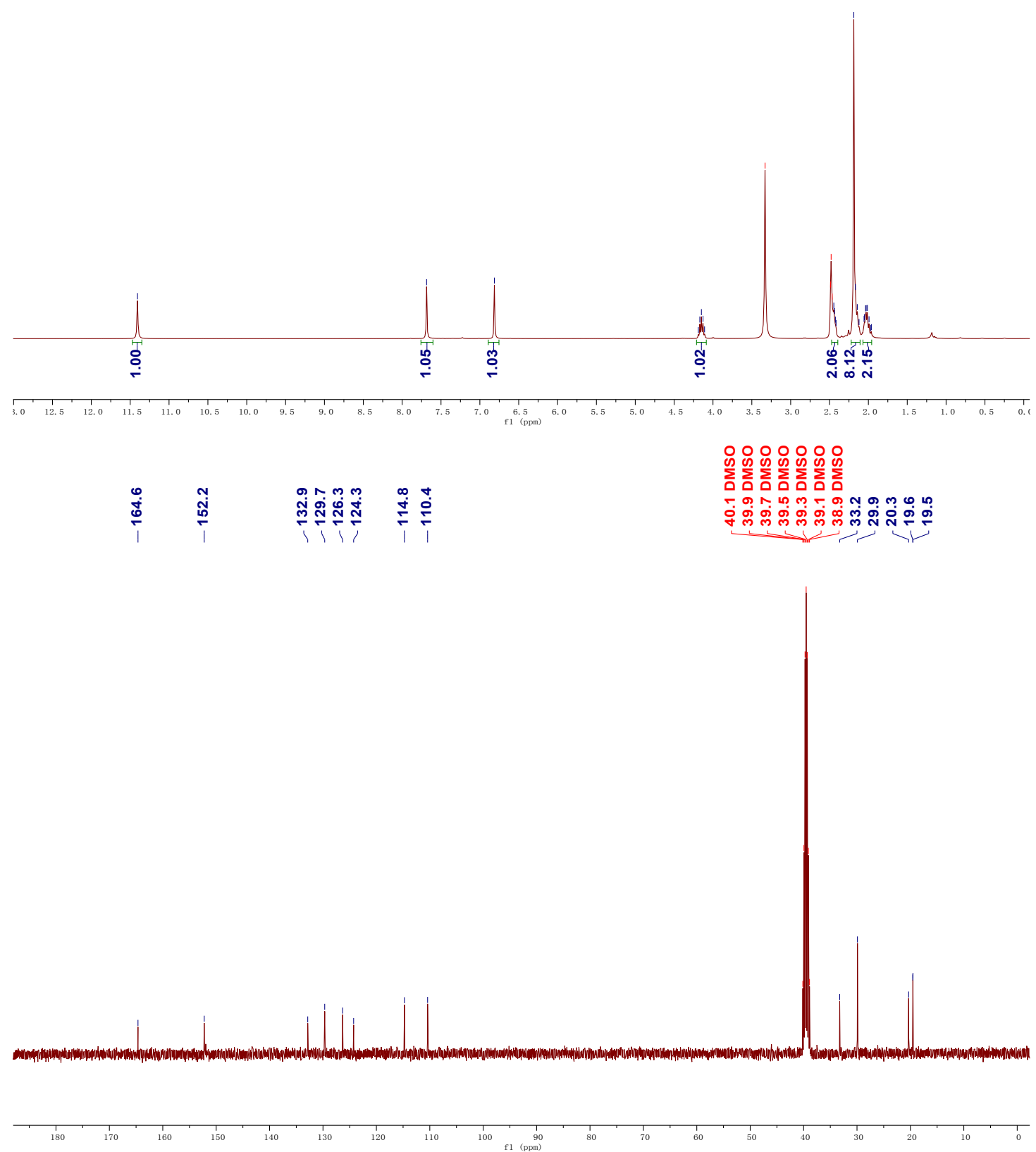
<smiles>Cc1cc2[nH]c(=O)n(C(=O)NCc3ccccc3)c2cc1C</smiles>
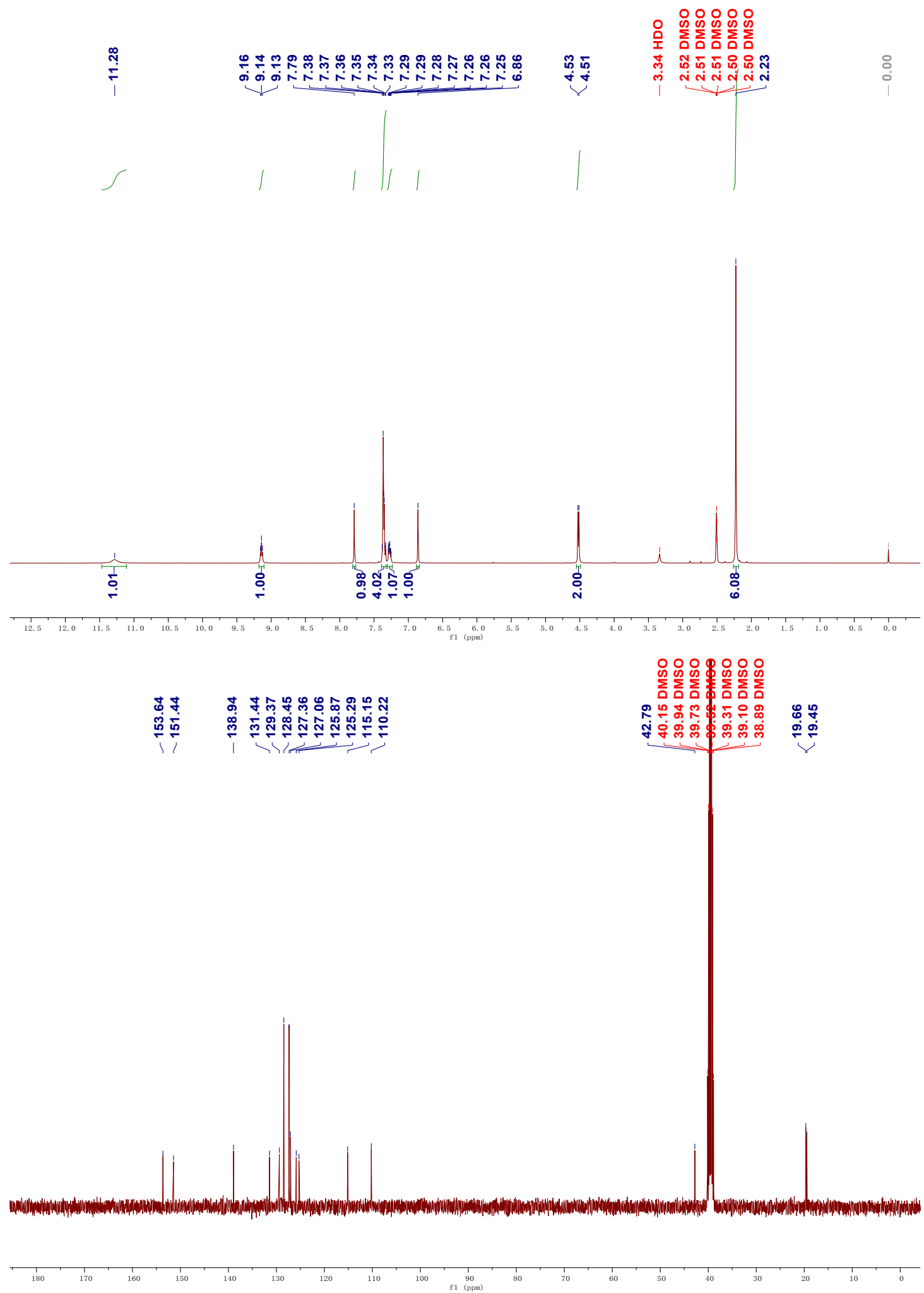


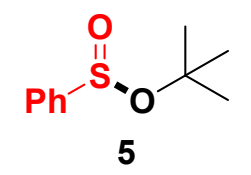

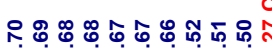

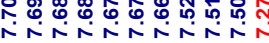

$\frac{m}{0}$
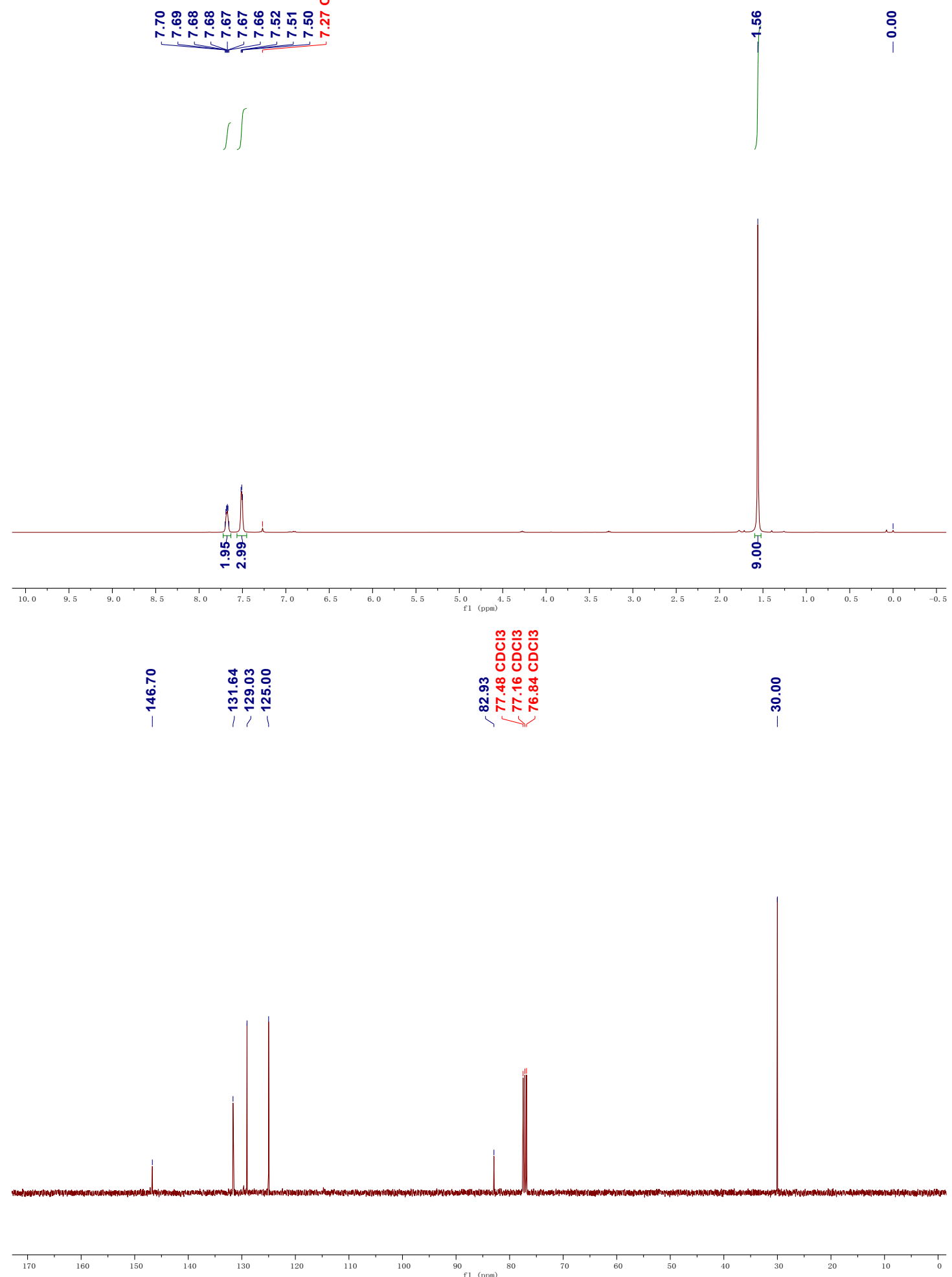


\section{References}

1. Ito, Y.; Ohnishi, A.; Ohsaki, H.; Murakami, M. Synthesis 1988, 1988, 714-715.

2. Du, B.; Wang, W.; Wang, Y.; Qi, Z.; Tian, J.; Zhou, J.; Wang, X.; Han, J.; Ma, J.; Pan, Y. Chem. Asian J. 2017, 13, 404-408. 